\title{
Kekulé Spiral Order at All Nonzero Integer Fillings in Twisted Bilayer Graphene
}

\author{
Y. H. Kwan $\odot,{ }^{1}$ G. Wagner, ${ }^{1}$ T. Soejima $\odot,{ }^{2}$ M. P. Zaletel, ${ }^{2,3}$ S. H. Simon, ${ }^{1}$ S. A. Parameswaran $\odot,{ }^{1}$ and N. Bultinck ${ }^{1,4}$ \\ ${ }^{1}$ Rudolf Peierls Centre for Theoretical Physics, University of Oxford, Oxford OX1 3PU, United Kingdom \\ ${ }^{2}$ Department of Physics, University of California, Berkeley, California 94720, USA \\ ${ }^{3}$ Materials Sciences Division, Lawrence Berkeley National Laboratory, Berkeley, California 94720, USA \\ ${ }^{4}$ Department of Physics, Ghent University, 9000 Ghent, Belgium
}

(Received 30 June 2021; accepted 8 November 2021; published 30 December 2021)

\begin{abstract}
We study magic angle graphene in the presence of both strain and particle-hole symmetry breaking due to nonlocal interlayer tunneling. We perform a self-consistent Hartree-Fock study that incorporates these effects alongside realistic interaction and substrate potentials and explore a comprehensive set of competing orders including those that break translational symmetry at arbitrary wave vectors. We find that at all nonzero integer fillings very small strains, comparable to those measured in scanning tunneling experiments, stabilize a fundamentally new type of time-reversal-symmetric and spatially nonuniform order. This order, which we dub the "incommensurate Kekulé spiral" (IKS) order, spontaneously breaks both the emergent valley-charge conservation and moiré translation symmetries but preserves a modified translation symmetry $\hat{T}^{\prime}$ - which simultaneously shifts the spatial coordinates and rotates the $U(1)$ angle which characterizes the spontaneous intervalley coherence. We discuss the phenomenological and microscopic properties of this order. We argue that our findings are consistent with all experimental observations reported so far, suggesting a unified explanation of the global phase diagram in terms of the IKS order.
\end{abstract}

DOI: 10.1103/PhysRevX.11.041063

Subject Areas: Condensed Matter Physics, Graphene, Strongly Correlated Materials

\section{INTRODUCTION}

The discovery of superconductivity proximate to correlated insulating behavior in a variety of graphene moiré heterostructures [1-4] has triggered intensive efforts to explore the phase structure of these highly tunable twodimensional materials. In the best-studied example, twisted bilayer graphene (TBG) tuned to a "magic angle" of approximately $1^{\circ}$, the enhancement of correlations is associated to the formation of extremely narrow bands due to the reconstruction of the electronic dispersion by the moiré superlattice. As noted by Bistritzer and MacDonald (BM) [5], this can be elegantly captured within a continuum model [6-8] where Dirac cones contributed by isolated graphene layers are coupled by interlayer tunneling modulated at the moiré scale. The BM model serves as a starting point for most theoretical studies of TBG. On combining the degeneracies corresponding to spin and microscopic valley indices with the Dirac structure enforced by exact and approximate symmetries of the moiré band structure,

Published by the American Physical Society under the terms of the Creative Commons Attribution 4.0 International license. Further distribution of this work must maintain attribution to the author(s) and the published article's title, journal citation, and DOI. the model reveals that the striking effects reported in experiments occur when the Fermi energy is tuned to lie within an octet of nearly flat bands that straddle charge neutrality. The phase structure of TBG then turns on the question of how electron correlations and other perturbations such as strain and substrate effects lift the approximate degeneracy within this subspace to select between a variety of competing ground states.

At first sight, the phase diagram of TBG resembles that of the cuprate high-temperature superconductors, with electrostatic gating playing the role of chemical doping. This prompted initial attempts to model correlation effects within a single-band Hubbard model for electronic states localized to a triangular moiré superlattice. Although this approach has proven fruitful in studying moiré heterostructures of $\mathrm{MoS}_{2}$ and other transition-metal dichalcogenides [9], its applicability to TBG is limited by the "fragile topology" of the BM bands $[10,11]$. The latter requires that the simplest tight-binding model that faithfully captures the symmetries of TBG involves a pair of crystallographically inequivalent Wannier orbitals centered on sites of a honeycomb lattice but with their charge densities peaked on a triangular lattice formed by the centers of its hexagons $[10,12,13]$. A corollary of this Wannier representation is that it implies a higher degree of itineracy than can be captured via a minimal honeycomb lattice Hubbard model with on-site repulsion and nearest-neighbor hopping. 
The utility of a Hubbard description is further challenged by the early experimental observation of a quantized anomalous Hall (QAH) resistance in TBG samples aligned with a hexagonal boron nitride $(h-\mathrm{BN})$ substrate, at an electron density corresponding to filling seven of the eight bands [14]. (In the convention where $\nu=0$ represents the filling at neutrality, this corresponds to $\nu=+3$.) Since this occurs in the absence of an external magnetic field, it indicates the spontaneous breaking of time-reversal symmetry (TRS). One explanation of this phenomenon invokes a compelling analogy to the other paradigmatic setting for strong correlations: the celebrated Landau levels (LLs) of an electron in a magnetic field. The TBG flat bands are endowed with nontrivial topology encoded in the winding of their Bloch functions across the moire Brillouin zone (mBZ); the inclusion of substrate potential triggers the opening of gaps between the moiré Dirac points (by breaking one of the protecting symmetries) and assigns nonzero Chern numbers to the bands, making them topologically equivalent to LLs. The absence of explicit TRS breaking is reflected in the assignment of equal and opposite Chern numbers to different valleys, which are exchanged by TRS. At $\nu=3$, electrons in the two remaining unfilled bands spontaneously polarize into one of the two valleys. This allows them to minimize their interaction energy by virtue of Pauli exclusion, leading to an insulator that spontaneously breaks TRS, with a quantized Hall resistivity of $\rho_{x y}=h / e^{2}$ protected by the charge gap. Recently, a similar QAH state was also observed at $\nu=1$ [15]. While the formation of such orbital Chern insulator states can, in principle, be captured within a Hubbard description [16], its close parallels to quantum Hall ferromagnetism (QHFM) [17-19] has motivated a distinct perspective, where TBG is viewed as a generalized multicomponent quantum Hall system. This naturally explains both the observed QAH response as well the propensity for insulating states at commensurate filling and motivates a sigma model description based on a hierarchy of perturbations around a "hidden" limit with $U(4) \times U(4)$ symmetry [20-22]. The QHFM picture receives further experimental support by the observed stabilization of QAH insulators [23] with Chern numbers $C= \pm 3, \pm 2, \pm 1$ at $\nu= \pm 1, \pm 2, \pm 3$ on applying a small out-of-plane magnetic field, even in the absence of substrate alignment $[15,24$ 27]. However, the TBG bands nevertheless retain features absent in LLs. For instance, their dispersion (though small) remains nonzero and is enhanced when particle-hole symmetry-breaking effects are incorporated-especially in the electron-doped regime-or upon inclusion of strain. Such effects are likely important in giving an accurate description of experimental samples. As a case in point, even at commensurate fillings some experiments [28] report gapless states or insulators with Chern numbers distinct from those of the noninteracting bands $[15,29,30]$. This suggests that departures from the flat band or QHFM limit are non-negligible and that the competition between itineracy and localization characteristic of Hubbard physics remains relevant to $\mathrm{TBG}$.

Given its enticing position at the intersection of two dominant themes of strong correlations, it is natural to conjecture that orders that are "natural" from both perspectives could be particularly robust candidate ground states in TBG. One example (and our focus here) is furnished by states with broken translation symmetry, which emerge in relatively well-understood limits of both the Hubbard and quantum Hall settings. The formation of charge and/or spin stripe order is believed to be a nearuniversal consequence of hole doping the cuprates away from commensurate filling: While purely on-site Hubbard repulsion favors phase separation, the inevitably present further-neighbor interactions frustrate this in favor of spatially ordered phases [31-43]. For similar energetic reasons, a variety of stripe and bubble phases are known to be competitive ground states in high Landau levels [4447]: Phase separation is driven by exchange physics and frustrated by Hartree contributions. As noted above, any Hubbard description of TBG must involve substantial further-neighbor interactions. Meanwhile, corrections to the flat band limit-particularly from strain and particlehole symmetry breaking - can significantly modify the Hartree potential, penalizing full occupation of the $\mathrm{mBZ}$. Since exchange interactions still favor insulating behavior, one resolution is to reconstruct the bands via finite-wavevector ordering. Thus, from both points of view, it appears that conditions in TBG might favor translationalsymmetry-breaking states over their competitors. Despite this, relatively little work focuses on this possibility, with rare exceptions [16,22,30,48-53], of which we highlight a few. References [16,51] identify a unidirectional charge density wave order that doubles the moiré unit cell in an interaction-only model with spin and valley degeneracy suppressed, but this does not appear to be energetically competitive with translationally invariant states in more realistic situations. More recently, a different perioddoubling stripe order stabilized by Hartree effects was invoked to explain the occurrence of commensurate-filling insulators whose Chern numbers depart from those expected from the naive QHFM picture [30] (although we suggest an alternative and possibly more natural translation-breaking order at these fillings below). Reference [50] focuses on the Dirac cones and classifies the possible flavor-breaking orders that can be connected to superconductivity via Wess-Zumino-Witten terms. Among the candidate normal-state orders are moiré density waves which couple the different minivalleys of TBG (see also Ref. [54] for similar discussion in twisted trilayer graphene) and, hence, break translation symmetry, but no microscopic studies have yet been performed to determine their energetic competitiveness. The nature of translation symmetry breaking differs from that in the state described in the 
subsequent sections, which retains the modified translation symmetry $\hat{T}^{\prime}$. Hence, it is not associated with charge or spin modulations between moiré $A A$ regions, unlike the moiré density waves. Reference [55] studies a valley spiral state in magnetically encapsulated TBG. This is a similar state to our proposed one, albeit the physical origin and the parameter space within which that state exists are quite different. [56] For completeness, we note that translational symmetry breaking has recently been observed in closely related twisted monolayer-bilayer graphene moiré heterostructures [57] and was proposed theoretically to explain insulating states observed in twisted bilayer $\mathrm{WSe}_{2}$ [58]. But, despite these previous works, to date there has been no systematic analysis of translational symmetry breaking in realistic TBG systems, and so the extent to which such symmetry breaking is a common phenomenon across the wide range of parameters relevant to experimental samples remains unclear.

In this work, we explore translational-symmetry-breaking order at commensurate integer fillings in TBG. Our analysis incorporates three experimentally important deviations from the Bistritzer-MacDonald limit—particle-hole symmetry breaking from nonlocal tunneling perturbations, a substrate potential, and uniaxial strain [59] - and also studies different interaction strengths and twist angles. In the balance of this introduction, we provide a digest of our main results, which also serves to signpost the organization of the remainder of this paper.

\section{A. Summary of results}

Our central finding, obtained from extensive HartreeFock simulations (discussed in Sec. II), is that, for modest (as little as $0.2 \%$ ) uniaxial strain and largely independent of all other parameters, the ground state of TBG at all nonzero integer fillings $\nu= \pm 1, \pm 2, \pm 3$ is a time-reversal invariant state that breaks superlattice translational symmetry [60] by modulating intervalley coherence at an incommensurate wave vector q. Since the intervalley coherence corresponds to a Kekule pattern at the microscopic graphene scale, one can view this order as a Kekulé pattern that rotates at the moiré scale with period $2 \pi /|\mathbf{q}|$ [Fig. 1]. We therefore dub this the "incommensurate Kekule spiral" (IKS) order. The IKS state is insulating at $\nu= \pm 2, \pm 3$ but does not show a charge gap at $\nu= \pm 1$. In contrast to its ubiquity at nonzero integer fillings, the IKS order is absent at charge neutrality, where we instead find the ground state for comparable strain to be a nematic semimetal, as identified in previous work [61]. An overview of all the phases found in HartreeFock is given in Fig. 2.

Modulations in the valley coherence are fundamental to the IKS state, which, hence, relies on the interplay between the moiré pattern and graphene-scale physics. This makes its properties and phenomenology distinct both from the previously studied period-2 stripe states in TBG [30] and from various stripe orders in other correlated systems.
It also differs in a few important ways from other proposed states with spatially modulated valley coherence in either TBG, twisted bilayer $\mathrm{WSe}_{2}$, or twisted monolayer-bilayer graphene $[48,49,58,62]$. First, IKS order generally occurs at an incommensurate wave vector, unlike the moiré density waves in Ref. [50]. Second, it does not rely on the presence of higher-order van Hove singularities or Fermi-surface nesting and, thus, has valley coherence over almost the entire mBZ. Third, the IKS state apparently requires a small, but nonzero, amount of strain to be stabilized against competing $\mathbf{q}=0$ orders. And finally, the IKS state is timereversal symmetric, unlike the state discussed for twisted monolayer-bilayer graphene in Ref. [62].

Although the IKS order parameter breaks the moiré translation $\hat{T}_{\mathbf{a}_{i}}$ and $U(1)_{V}$ valley symmetries, it preserves $\hat{T}_{\mathbf{a}_{i}}^{\prime}=\hat{T}_{\mathbf{a}_{i}} e^{i \mathbf{q} \cdot \mathbf{a}_{i} \tau_{z} / 2}$ (where $\mathbf{a}_{i}$ are moiré lattice vectors and $\tau_{\mu}$ denote a set of Pauli matrices acting in valley space). Performing a valley-dependent gauge transformation, therefore, yields eigenstates that satisfy a generalized Bloch theorem. This transformation, which amounts to shifting the dispersion in the valleys by $\pm \mathbf{q} / 2$ in the $\mathrm{mBZ}$, allows us to label electronic states in the IKS state by an analog of the crystal momentum associated with $\hat{T}_{\mathbf{a}_{i}}^{\prime}$ (rather than $\hat{T}_{\mathbf{a}_{i}}$ ), without folding the $\mathrm{mBZ}$. This shifted-mBZ perspective yields a simple yet quantitatively accurate ansatz for the HF projector in the IKS state, which, in turn, allows us to link the origin of the order to features of the interaction-renormalized BM bands. We can also use the preservation of $\hat{T}_{\mathbf{a}_{i}}^{\prime}$ to derive a modified Lieb-SchulzMattis theorem that forces gapped IKS order to appear only at integer fillings unless it triggers subsidiary topological or symmetry-breaking order. This explains why, despite involving a modulation that is incommensurate with the moiré lattice, the IKS insulator is tied to densities that are commensurate with it.

As we show below, the precise magnitude and direction of the spiral wave vector $\mathbf{q}$ are controlled by details of the dispersion of the interaction-renormalized bands in the $\mathrm{mBZ}$. The former is roughly $1 / 3$ of the width of the $\mathrm{mBZ}$, while the latter is approximately aligned along a moiré crystallographic axis, but the energy cost for varying away from these values (the "stiffness" of the IKS order) is quite small. In a sense, one can view this softness as being partly responsible for the robustness of the IKS state, since it allows the ordered state to respond to variations in external parameters such as substrate potential, twist angle, or interaction strength by adjusting $\mathbf{q}$ while keeping its other properties essentially unchanged.

The above results are obtained in Sec. III by focusing initially on fillings $\nu= \pm 2$ where the IKS order is especially simple to describe. We broaden the scope of our analysis in Sec. IV to also consider $\nu= \pm 1$ and $\nu= \pm 3$, which differ primarily in the spin structure and the stability of IKS order against competing phases. 
Interestingly, the time-reversal-symmetric IKS order provides a way to obtain insulating states with zero Chern number at the odd integer fillings. Such states are difficult to obtain within the QHFM formalism and are observed experimentally in Refs. $[3,15]$. In Sec. V, we show that the $\nu=-3$ state provides a "basis spiral" that serves as a building block for IKS states at other fillings, in a manner that we make quantitatively precise.

We derive a Landau-Ginzburg theory of IKS order in Sec. VI and use this to link the circular (elliptical) nature of the spiral order in valley coherence to the absence (presence) of subsidiary charge density modulations. We also consider the response of the IKS state to quenched disorder (Sec. VII) and thermal fluctuations (Sec. VIII). A key point is that, although disorder on the microscopic graphene scale can couple to the Kekule distortion as a random field, this is suppressed in powers of the ratio of the graphene and moiré lattice constants, scaling as $\theta^{4}$ for small twist angles $\theta$. Consequently, the dominant disorder fluctuations are those that occur on the moiré scale. This justifies our assignment of a definite Kekulé order to each $A A$-stacking region that defines a superlattice "site." Thermal effects are more delicate, owing to a rich set of symmetries broken by the IKS state: namely, valley- $U(1)$, superlattice translation, and threefold rotation. Since the superlattice translation is broken solely by valley- $U(1)$ charged operators, longrange order in both is absent at any temperature $T>0$ and is replaced by algebraic correlations, which, in turn, become exponential decay above a Berezinskii-KosterlitzThouless transition temperature $T_{\mathrm{BKT}}$ at which vortices in the valley order proliferate. In contrast, the rotational symmetry breaking persists as true long-range $T>0$ order, so that (ignoring explicit symmetry breaking from strain) the finite-temperature IKS state has a nematic order up to a finite Ising transition at $T_{N}$. Depending on the ratio of $T_{N}$ to $T_{\mathrm{BKT}}$, we can have a variety of different thermal melting scenarios based on which order is lost first, though the precise details are subtle and may depend on the ability of the superlattice to pin $\mathbf{q}$ to a commensurate value (which is likely weak). The relevant scales for $T_{N}$ and $T_{\mathrm{BKT}}$ are similar, are set by the IKS stiffness, and are approximately $7 \mathrm{~K}$, which is comparable with the experimental temperature scales at which gapped insulators are observed.

We emphasize (Sec. IX) that our results closely match current experiments: most notably, through the absence of spin polarization in the $\nu= \pm 2$ IKS, the relatively greater robustness (as measured by the charge gap) of insulating states on the electron-doped side $(\nu>0)$, and the ability of the IKS to "reset" the Chern number to zero at odd integer fillings. The IKS order is a nematic at finite temperature. Thus, we expect the orientational symmetry-breaking effects of strain to be heavily enhanced in the IKS state, making it a natural proximate order to the reported nematic superconductors near $\nu=-2,-3$. A subset of experiments find correlated insulators at all integer fillings except at neutrality, where they see evidence of a gapless state, and $\nu= \pm 1$, where weak insulating peaks have been observed [4]. This is readily reconciled with our results_-since (see Fig. 2) we find IKS order for all integer $\nu$ except $\nu=0$-if we assume that the relevant experimental samples are subject to a small amount of heterostrain. (This is a relatively mild assumption given the weak strain needed to stabilize the IKS state and the $\nu=0$ nematic semimetal.) Direct verification of IKS order is a more challenging goal. Owing to the unusual nature of IKS states, the translational symmetry breaking is invisible to valley-diagonal observables, and a Landau-Ginzburg analysis reveals that the circular spiral order does not trigger a parasitic chargedensity wave order. Nevertheless, since intervalley coherence necessarily triggers spatial order on the graphene scale, the associated Kekule distortion should be apparent, e.g., in the locally $A A$ regions of the superlattice, but will be modulated at the moiré scale. This order can, in principle, be detected via scanning probes, though the sensitivity required may be difficult to achieve in the very near term. We close with a discussion of future directions motivated by this work, in Sec. X. We provide details of numerical simulations and additional analysis in five technical Appendixes in Supplemental Material [63].

\section{MODEL AND NUMERICAL TECHNIQUES}

In this section, we discuss the interacting BistritzerMacDonald (BM) model in the presence of strain, substrate potential, and nonlocal tunneling (NLT) and describe our HF calculations. Further details can be found in the Appendixes [63].

We begin with the standard single-particle BM model [5] describing the band structure of two graphene layers $l=1,2$ stacked with a relative twist $\theta$ near the magic angle. For concreteness, we orient the coordinate system such that the untwisted monolayer Dirac points lie at $\mathbf{k}= \pm K_{D} \hat{x}$. The interlayer coupling, which is modulated by the moiré pattern, is parametrized by sublattice-dependent hopping constants $w_{A A}=82.5 \mathrm{meV}$ and $w_{A B}=110 \mathrm{meV}$. The presence of different coupling constants arises from corrugation effects $[64,65]$ that increase the interlayer spacing in $A A$-stacking regions compared to $A B$ (Bernal) regions.

Throughout this work, we fix $\theta=1.08^{\circ}$, unless stated otherwise. Including both spins and valleys (with corresponding Pauli matrices $s_{\mu}$ and $\tau_{\mu}$, respectively), the BM Hamiltonian has eight central bands near the neutrality point with narrow bandwidth approximately $5 \mathrm{meV}$ and large gaps approximately $30 \mathrm{meV}$ to the remote bands [Fig. 1(b)]. The central band wave functions are concentrated at the $A A$-stacked regions [Fig. 1(a)], which form the moiré lattice. The model possesses (spinless) TRS $\hat{\mathcal{T}}=\tau_{x} \hat{\mathcal{K}}$ (where $\hat{\mathcal{K}}$ denotes complex conjugation) and enjoys emergent $D_{6}$ and valley-charge conservation $\left[U(1)_{V}\right]$ symmetries and an approximate particle-hole symmetry (PHS) 
(a)

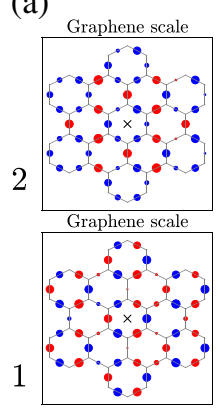

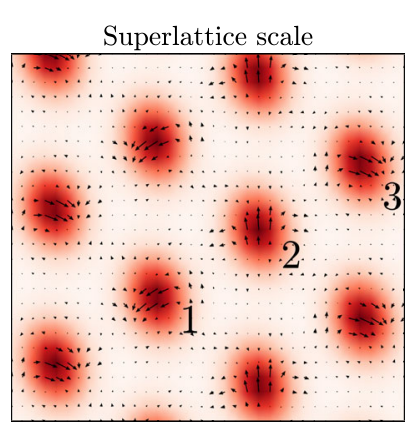

(b)

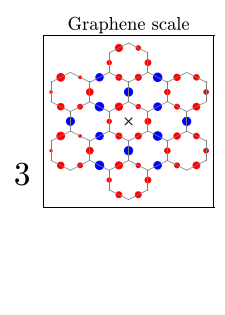

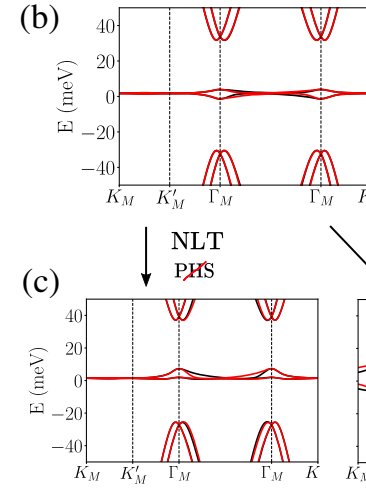

(c) (d)
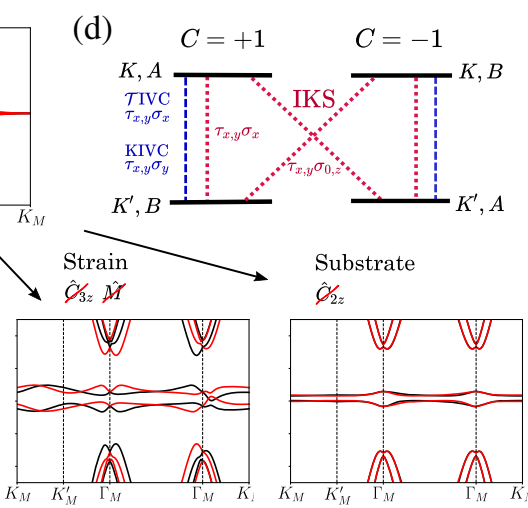

Substrate

$\hat{\mathscr{C}} / 2$

\section{.}

FIG. 1. (a) Real-space picture of an IKS state with $\mathbf{q}=\mathbf{G}_{1} / 3$. The color plot on the superlattice scale shows the charge density, with dark spots corresponding to $A A$ regions. Black arrows represent the complex IVC order parameter $\sim\left\langle\tau_{x} \sigma_{x}\right\rangle+i\left\langle\tau_{y} \sigma_{x}\right\rangle$. For each of the three inequivalent $A A$ regions, the expectation value of $c_{A}^{\dagger} c_{B}+c_{B}^{\dagger} c_{A}$ on the microscopic graphene bonds is shown. Blue (red) dots correspond to positive (negative) expectation values, and the center of the $A A$ region is marked with a black cross. The different inequivalent $A A$ regions have different approximate $\sqrt{3} \times \sqrt{3}$ Kekulé-like patterns on the graphene scale. (b) The red (black) line shows $\mathrm{BM}$ band structure along a cut in the $\mathrm{mBZ}$ for valley $K\left(K^{\prime}\right)$. (c) The presence of nonlocal tunneling, strain, and substrate potential breaks various symmetries and affects the dispersion. (d) If these single-particle perturbations are weak, the interacting model has an approximate $U(4)_{C=1} \times U(4)_{C=-1}$ symmetry. Dashed and dotted lines indicate the channel of intervalley coherence that generically occurs for $U(1)_{V}$-breaking phases. $\tau_{x, y}$ denotes any valley-off-diagonal components.

$[10,11,66]$. A related antiunitary symmetry $\hat{\mathcal{T}}^{\prime}=\tau_{y} \hat{\mathcal{K}}$ can be defined, which is a signature of the Kramers intervalley coherent (KIVC) phase [20] to be reviewed below. The relevant spatial symmetries of the single-valley BM Hamiltonian are $\hat{C}_{2 z} \hat{\mathcal{T}}, \hat{C}_{3 z}$, and $\hat{M}$, where the latter corresponds to an in-plane twofold rotation around the $x$ axis which interchanges the two layers. Spin-orbit coupling is neglected, resulting in a total $U(2)_{K} \times U(2)_{K^{\prime}}$ flavor symmetry.

\section{A. Chern basis and effect of a substrate potential}

The central bands bear a remarkable resemblance to zero Landau levels in opposite fields (an analogy which is sharpened in the chiral limit $w_{A A}=0$ [67]). For a given spin or valley, we can take advantage of the weak dispersion to rotate the pair of central BM bands into a $C= \pm 1$ Chern basis by diagonalizing the sublattice operator $\sigma_{z}$
[20]. Each band carries substantial sublattice polarization (tending to \pm 1 in the chiral limit), and, hence, we use $\sigma$ to also refer to this basis. The Chern number of each of the degenerate bands is tied to its valley according to $C=\sigma_{z} \tau_{z}$ $[10,20,68]$. (Note that the "Chern basis" defined by a definite value of $C$ does not coincide with the eigenbasis of the single-particle dispersion in the absence of a substrate potential.)

Alignment of TBG with the $h$-BN substrate directly couples to the Chern basis via a sublattice mass $\sim \sigma_{z}$ with strength $\Delta \simeq 10-20 \mathrm{meV}$ [69-71] (we ignore the additional moiré potential coming from the mismatch between the graphene and $h$-BN lattice constants, although it has recently been argued to be important for explaining some of the experimental features [72,73]) and violates $\hat{C}_{2 z}$ and $\hat{M}$. The breaking of $\hat{C}_{2 z} \hat{\mathcal{T}}$ gaps the Dirac points [Fig. 1(c)], resulting in the formation of Chern bands. Polarization into a subset of these Chern bands (akin to quantum Hall
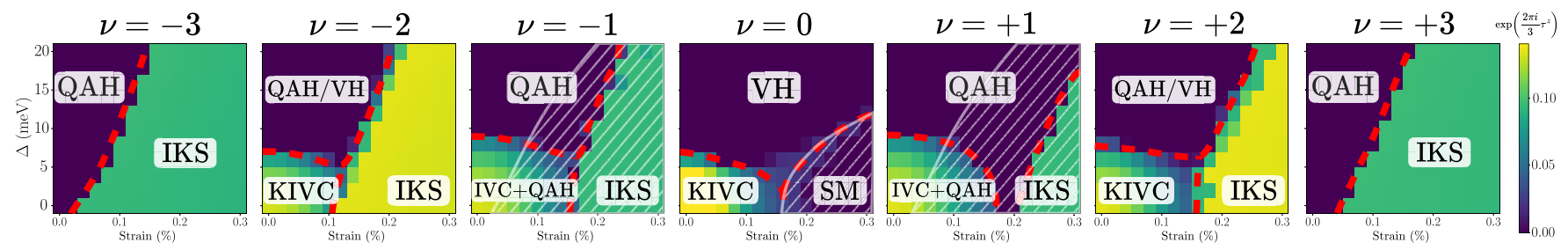

FIG. 2. Summary of phases found in self-consistent Hartree-Fock at all integer fillings $\nu$ for different heterostrains and substrate potentials $\Delta$, with nonlocal tunneling included. Color plot diagnoses IVC order [dark blue indicates unbroken $U(1)_{V}$ symmetry]. Dashed red lines indicate approximate phase boundaries, and hatched areas denote absence of a charge gap. Properties of each phase are tabulated in Table I. The phase diagrams here are presented in greater detail in Figs. 3 and 6. (IKS, incommensurate Kekulé spiral; QAH, quantized anomalous Hall state; KIVC, Kramers intervalley coherent state; VH, valley Hall state; IVC, intervalley coherence; SM, semimetal.). 
ferromagnetism) is believed to explain the observation of the anomalous Hall $(\mathrm{AH})$ effect at $\nu=+3$ in aligned samples [14,29]. The substrate-reconstructed central bands are also used as a starting point for constructing more exotic correlated states [74-79].

\section{B. Strain effects}

Uniaxial strain of strength $\epsilon=0.1 \%-0.7 \%$ is observed in many TBG samples using STM or STS [80-82]. At charge neutrality, this small strain is believed to be an important driving force behind the weakening of symmetry-broken insulators found in numerics at zero strain in favor of semimetallic phases $[51,61,83]$. In the context of van der Waals homobilayers, it is useful to distinguish between homostrain, where strain is applied identically to both layers, and heterostrain, where the layers are strained independently. Since homostrain, to first order, does not account for the experimentally observed distortion of the moiré lattice and has a substantially smaller impact on the electronic structure [84], we focus on heterostrain [61,85], which is also believed to be experimentally relevant. The moiré lattice vectors $\mathbf{a}_{1,2}$ are deformed depending on the value of the strain ratio $\epsilon$ and strain angle $\varphi$ with respect to the $x$ axis. The orthogonal direction is also stretched or compressed due to the Poisson ratio $\simeq 0.16$ [86]. To first order in $\epsilon$ and $\theta$, the twist angle is unaffected. The BM model is modified by taking into account the deformed superlattice basis vectors, as well as adding an effective layer-dependent vector potential $\mathbf{A}_{l}$ (similar to the orbital effect of an in-plane magnetic field [87]). Strain preserves $\hat{C}_{2 z}$ but breaks $\hat{C}_{3 z}$ and $\hat{M}$. Hence, the Dirac points remain intact but are unpinned from the $K_{M}$ and $K_{M}^{\prime}$ points and migrate toward the $\mathrm{mBZ}$ center [85]. The Dirac points also separate in energy, leading to Fermi pockets at charge neutrality, and the overall bandwidth of the central bands increases dramatically [see Fig. 1(c)].

\section{Nonlocal tunneling and breaking of particle-hole symmetry}

The standard BM Hamiltonian obeys PHS very wellthe only violations come from small twists in the Dirac cone kinetic terms which are suppressed in $\theta[11,66]$. However, many experiments show pronounced electronhole asymmetry $[2,3,14,29,30,88,89]$, with stronger superconductors on the hole side and more robust insulators on the electron side. We model this PHS breaking by augmenting the BM model with a nonlocal interlayer tunneling term [65,90-92]. Consistent with density functional theory calculations, the effect of this term is to make the conduction bands more dispersive than the valence bands [65,90] [Fig. 1(c)]. We use the form of NLT motivated in Ref. [90] and choose values $\lambda_{2}=2 \lambda_{1}=0.18 \mathrm{eV} \AA, \lambda_{3}=0$ (see Supplemental Appendix A for definitions and a discussion on combining NLT and strain [63]).

\section{Hartree-Fock procedure}

We perform self-consistent HF calculations on the singleparticle Hamiltonian with dual-gate screened Coulomb interactions $V(q)=\left(e^{2} / 2 \epsilon_{0} \epsilon_{r} q\right) \tanh q d$, where the screening length $d=25 \mathrm{~nm}$ and relative permittivity $\epsilon_{r}=10$. We neglect terms which scatter electrons between the valleys, as they are suppressed for small $\theta$. Along with electron-phonon scattering, such "intervalley Hund's couplings" would weakly break the $U(2)_{K} \times U(2)_{K^{\prime}}$ symmetry [20]. In order to avoid double-counting interaction effects, we subtract off a density matrix corresponding to decoupled graphene layers at charge neutrality $[20,93]$. The results shown the main text are obtained by considering only the central bands as active, with the remote valence (conduction) bands frozen to be filled (empty). However, we check that increasing the number of active bands does not lead to a qualitative change in the results and mainly leads to a decrease in the band gap and a slight shift in the phase boundaries. For more details on the effects of adding more active bands, we refer to Appendix D [63].

In our numerical simulations, we consider completely general moiré translation symmetry-breaking Slater determinants with single-particle density matrices of the general form

$$
\left\langle\hat{c}_{\mathbf{k} \tau a s}^{\dagger} \hat{c}_{\mathbf{k}^{\prime} \tau^{\prime} a^{\prime} s^{\prime}}\right\rangle=P_{\mathbf{k} \tau a s ; \mathbf{k}^{\prime} \tau^{\prime} a^{\prime} s^{\prime}},
$$

satisfying $\operatorname{Tr} P=(\nu+4) N_{1} N_{2}$, where $N=N_{1} N_{2}$ is the number of moiré unit cells and $a$ and $a^{\prime}$ are BM band indices. We use periodic boundary conditions in both directions, which leads to discrete values for the allowed momenta, viz. $\mathbf{k}, \mathbf{k}^{\prime}=\left(n_{1} / N_{1}\right) \mathbf{G}_{1}+\left(n_{2} / N_{2}\right) \mathbf{G}_{2}$, with $\mathbf{G}_{i}$ the moiré reciprocal lattice vectors.

For most calculations, we enforce collinearity of the spins and accelerate convergence using the optimal damping algorithm [94,95]. All the states found in our HF simulations (discussed in detail in the following sections) contain at most a single wave-vector modulation, meaning that $P_{\mathbf{k} \tau a s ; \mathbf{k}^{\prime} \tau^{\prime} a^{\prime} s^{\prime}} \neq 0$ only for $\mathbf{k}^{\prime}=\mathbf{k}, \mathbf{k}+\mathbf{q}, \mathbf{k}-\mathbf{q}$. As detailed below, whenever translational symmetry breaking occurs, we find that the finite-q component of the projector $P$ is entirely off diagonal in valley space.

\section{SPIN-UNPOLARIZED KEKULÉ SPIRALS AT $\nu= \pm 2$}

We now explore how the trio of realistic modifications to the BM model introduced above-namely, substrate effects, strain, and particle-hole symmetry breaking-stabilize phases that compete with those previously proposed for the idealized situation where these modifications are absent $[20,22,51,83,91,93,96-104]$. In this section, we first focus on fillings $\nu= \pm 2$, as the phenomenology of the translationalsymmetry-breaking states is especially clear here. 


\section{A. Numerical Hartree-Fock results}

Figure 3(a) presents the HF phase diagrams at $\nu= \pm 2$ in the presence of NLT as a function of both strain and substrate potential. The color scale diagnoses the magnitude of $U(1)_{V}$ breaking. Without symmetry-breaking perturbations, the lowest energy state is the $\hat{\mathcal{T}}^{\prime}$-symmetric KIVC state [20] (see also Ref. [98]). It consists of a filled intervalley coherent (IVC) band in each Chern sector and can be succinctly described by ordering of $\tau_{x, y} \sigma_{y}$, where $\tau_{x, y}$ denotes any offdiagonal component in valley space [Fig. 1(d)]. The absence of coherence between opposite Chern sectors sidesteps the energy penalty induced by vortices in the order parameter that would be topologically required for other IVC candidates [70]. At $U(2)_{K} \times U(2)_{K^{\prime}}$ level, there is a manifold of degenerate states with different spin polarizations (with maximum $2 \mu_{B}$ spin moment per moiré cell), but intervalley Hund's perturbations will lift this degeneracy.

At a finite substrate potential strength, the optimal state becomes a sublattice-polarized $U(1)_{V}$-symmetric ferromagnet, which can be either the QAH state $\sim \sigma_{z} \tau_{z}$ or the valley Hall state $(\mathrm{VH}) \sim \sigma_{z}$. These are exactly degenerate at HF level, since the VH state is obtained by applying $\hat{\mathcal{T}}$ on one spin component of the QAH state.
Along the strain axis, we find a first-order transition to a novel phase, which we dub the incommensurate Kekulé spiral (IKS) state, at an experimentally relevant strain ratio of $\epsilon \sim 0.1 \%-0.2 \%$ [Fig. 3(b)]. The main characteristic of the IKS state is the breaking of moiré translation symmetry at a single wave vector $\mathbf{q}$. The translation breaking occurs entirely in the intervalley channel and is clearly identified in HF by the nonvanishing of the following density matrix elements in the sublattice-polarized basis:

$$
\left\langle\hat{c}_{\mathbf{k}+\mathbf{q}, \tau=+, \sigma^{\dagger}}^{\dagger} \hat{c}_{\mathbf{k}, \tau^{\prime}=-, \sigma^{\prime}}\right\rangle \sim f_{\sigma, \sigma^{\prime}}(\mathbf{k}),
$$

where spin labels are omitted. Importantly, the IVC occurs at a single $\mathbf{q}$, leading to a circular intervalley coherent spiral of definite handedness, as there is no symmetry relating the spiral we find in HF to the analogous spiral at -q [Fig. 3(c)]. The IKS state also preserves TRS $\hat{\mathcal{T}}$ and has zero total spin and valley polarization (Table I). Since the spins within each valley are also unpolarized, inclusion of intervalley Hund's coupling does not lead to qualitative changes.

The IKS order persists for fairly large substrate potential strengths. This is expected, since the intervalley coherence is flexible enough to polarize onto one sublattice, as (a)
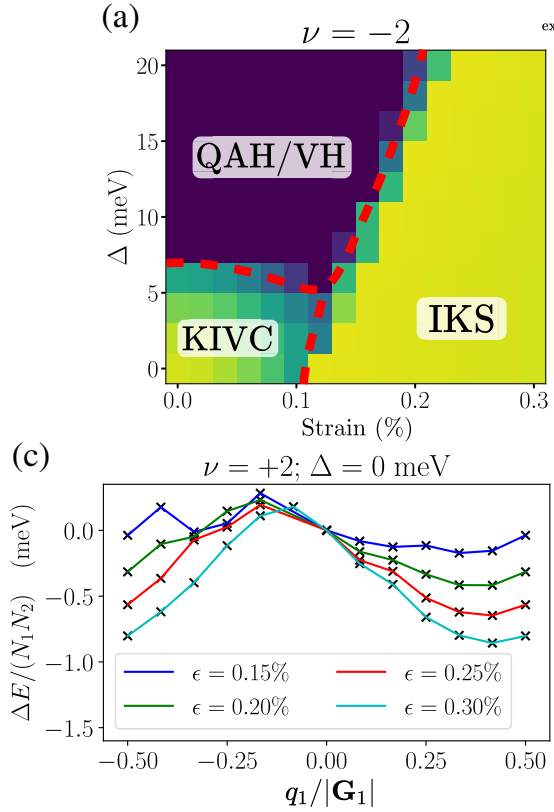

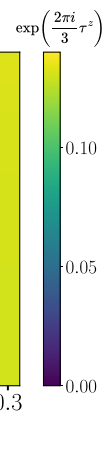

(d)
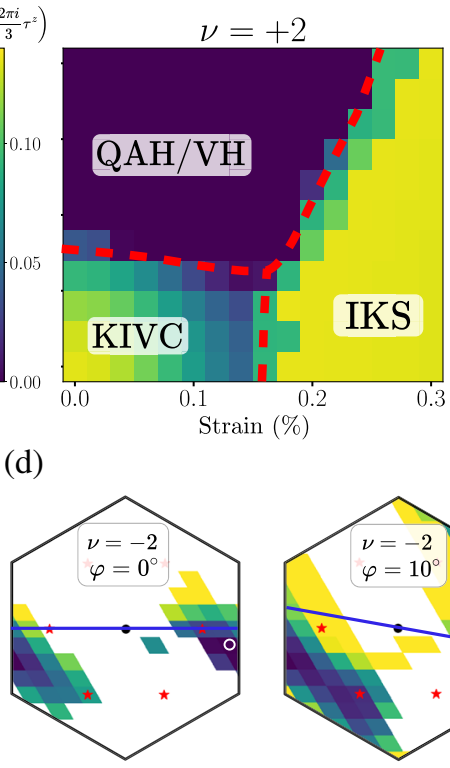

(b)

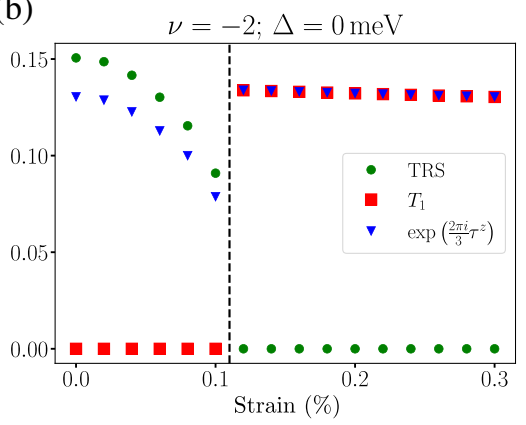

FIG. 3. (a) $\nu= \pm 2$ strain-substrate HF phase diagram (enlarged view of panels from Fig. 2). Strain is along the $x$ axis $\left(\varphi=0^{\circ}\right)$, and translational symmetry breaking is restricted to $\mathbf{q}=\mathbf{G}_{1} / 3$. The color plot diagnoses IVC order by taking the Frobenius norm of the difference of the density matrix after acting with $e^{(2 \pi i / 3) \tau_{z}}$. Dashed red lines indicate approximate phase boundaries. Particle-hole breaking is introduced via nonlocal tunneling (NLT). System size is $12 \times 12$, and only central bands are active. (b) Phase transition between KIVC and IKS states along the strain axis of (a) at $\nu=-2$. In the IKS phase, combined $U_{V}(1)$ rotation by $2 \pi / 3$ and real-space translation by $\mathbf{a}_{1}$ is a symmetry. (c) IKS energy relative to the lowest translation-symmetric state for different strains and spiral wave vectors $q$ along $\mathbf{G}_{1}$. NLT is not included. (d) Relative energies of IKS states for different strain angles $\varphi$ (blue axis) and enforced symmetry-breaking wave vectors $\mathbf{q}$ [see Eq. (2)]. Red stars denote q's corresponding to period tripling along the moiré axes, and white circles denote minimum energy wave vectors $\mathbf{q}_{0}$. Non-IKS states that converge to higher energies are discarded. Strain is $0.2 \%$, and NLT is not included. Data points are transformed to fit on a hexagonal $\mathrm{mBZ}$. 
TABLE I. Symmetries and order parameters of HF phases at integer fillings $\nu$. Spin (valley) polarization indicates the number imbalance of $\uparrow$ vs $\downarrow$ ( $K$ vs $\left.K^{\prime}\right)$ electrons per moiré unit cell. The spin quantization axis is arbitrary due to $S U(2)_{S}$ symmetry, and an asterisk indicates a degenerate manifold obtained by performing a valley-dependent spin rotation. $\tau_{\mu}$ are Pauli matrices in valley space, and $\hat{\mathcal{K}}$ is complex conjugation. IKS phases break a subset of moiré translations $\hat{T}_{\mathbf{a}_{i}}$ but preserve the combined valley-rotation + translation symmetry $\hat{T}_{\mathbf{a}_{i}} e^{i \mathbf{q} \cdot \mathbf{a}_{i} \tau_{z} / 2}$, where $\mathbf{q}$ is the IKS wave vector.

\begin{tabular}{|c|c|c|c|c|c|c|c|c|c|}
\hline Phase & $|\nu|$ & Spin polarization & Valley polarization & $U(1)_{V}$ & $\hat{\mathcal{T}}=\tau_{x} \hat{\mathcal{K}}$ & $\hat{\mathcal{T}}^{\prime}=\tau_{y} \hat{\mathcal{K}}$ & $\hat{T}_{\mathbf{a}_{i}}$ & $\hat{T}_{\mathbf{a}_{i}} e^{i \mathbf{q} \cdot \mathbf{a}_{i} \tau_{z} / 2}$ & $|C|$ \\
\hline \multirow[t]{3}{*}{ IKS } & 1 & $*$ & 0 & $x$ & $\checkmark$ & $x$ & $x$ & $\checkmark$ & 0 \\
\hline & 2 & 0 & 0 & $x$ & $\checkmark$ & $x$ & $x$ & $\checkmark$ & 0 \\
\hline & 3 & $*$ & 0 & $x$ & $\checkmark$ & $x$ & $x$ & $\checkmark$ & 0 \\
\hline \multirow[t]{3}{*}{ QAH } & 1 & 1 & 1 & $\checkmark$ & $x$ & $x$ & $\checkmark$ & & 1 \\
\hline & 2 & 0 & 2 & $\checkmark$ & $x$ & $x$ & $\checkmark$ & & 2 \\
\hline & 3 & 1 & 1 & $\checkmark$ & $x$ & $x$ & $\checkmark$ & & 1 \\
\hline \multirow[t]{2}{*}{ KIVC } & 0 & 0 & 0 & $x$ & $x$ & $\checkmark$ & $\checkmark$ & & 0 \\
\hline & 2 & $*$ & 0 & $x$ & $x$ & $\checkmark$ & $\checkmark$ & & 0 \\
\hline \multirow[t]{2}{*}{$\mathrm{VH}$} & 0 & 0 & 0 & $\checkmark$ & $\checkmark$ & $\checkmark$ & $\checkmark$ & & 0 \\
\hline & 2 & $*$ & 0 & $\checkmark$ & $\checkmark$ & $\checkmark$ & $\checkmark$ & & 0 \\
\hline $\mathrm{QAH}+\mathrm{IVC}$ & 1 & 1 & 1 & $x$ & $x$ & $x$ & $\checkmark$ & & 1 \\
\hline SM & 0 & 0 & 0 & $\checkmark$ & $\checkmark$ & $\checkmark$ & $\checkmark$ & & 0 \\
\hline
\end{tabular}

evidenced from the fairly constant magnitude of IVC throughout the phase. On the other hand, the KIVC is progressively weakened under increasing sublattice potential and gives way to $U(1)_{V}$-preserving ferromagnets, since its mechanism relies on intersublattice coherence [20]. The strong PHS-breaking effect of NLT manifests in the shifted phase boundaries between $\nu=-2$ and +2 . Furthermore, the zero-substrate band gaps (of the order of $10 \mathrm{meV}$ ) of both the KIVC and IKS phases are larger on the electron side than the hole side by $10 \%-30 \%$, which is consistent with the experimental trend of more robust insulators at positive fillings.

The numerical phase diagram in Fig. 3(a) is constructed by restricting to a strain angle $\varphi=0$ and period-tripling order along $\mathbf{G}_{1}=\left|\mathbf{G}_{1}\right| \hat{x}$. However, when we relax this requirement, we find that the IKS phase actually consists of a family of spirals which differ only in their ordering wave vector $\mathbf{q}$ and are close in energy. Figure 3(c) plots the IKS energy relative to the best translation-symmetric state, as a function of $q_{1}$ ( $q_{2}$ is fixed to 0 , so translation symmetry is maintained along $\mathbf{a}_{2}$ ). The ideal wave vector $\mathbf{q}_{0}$ is slightly greater than $1 / 3$ of the $\mathrm{mBZ}$ and evolves weakly with strain magnitude. Hence, the spiral ordering generically occurs at an incommensurate $\mathbf{q}_{0}$ [see also Fig. 6(b)].

Figure 3(d), where we fully relax the constraints on the wave vector $\mathbf{q}$ in our HF calculations, reveals that the dispersion about $\mathbf{q}_{0}$ is very soft in both directions. Note that we check that, even without enforcing a particular q, the HF still converges only to a single-q state. We find the energy density of the IKS state to have a term of the form $\left(\rho_{s} / 2\right)\left(\mathbf{q}-\mathbf{q}_{0}\right)^{2}$, from which we estimate the wave-vector stiffness to be $\rho_{s} \sim 0.4 \mathrm{meV}$, without strong spatial anisotropy. In Fig. 3(d), we show that, as the strain angle $\varphi$ rotates, $\mathbf{q}_{0}$ also changes but appears to have roughly constant magnitude and predominantly lies near a moiré crystallographic axis. Figures 3(c) and 3(d) are computed without NLT; including NLT does not affect the qualitative features of these plots.

Before concluding the discussion of our numerical HF results, we want to point out the following subtlety. In the absence of $\hat{C}_{3 z}$ symmetry (which is broken by strain), the $\Gamma$ point of the single-valley BM model is no longer a highsymmetry point. From this, one might conclude that the choice of $\Gamma$ in one of the two valleys becomes arbitrary (the $\Gamma$ point in the other valley is still fixed by either $\hat{C}_{2 z}$ or $\hat{\mathcal{T}}$ ). Making a different choice for $\Gamma$ does not go without consequences for the IKS state, as this changes the wave vector $\mathbf{q}$ at which the intervalley coherence occurs. For commensurate twist angles, however, there is a preferred $\Gamma$ point in the mBZ even in the absence of $\hat{C}_{3 z}$-namely, it is the point that should fold on top of the $\Gamma$ point of the monolayer graphene BZ (which is fixed by $\hat{C}_{2 z}$ or $\hat{\mathcal{T}}$ ). From this it is clear that the wave vector $\mathbf{q}$ is well defined for commensurate twist angles and that the corresponding superlattice translation symmetry is unambiguously broken. In our numerical simulations at incommensurate twist angles, we always use the same choice for the $\mathrm{mBZ} \Gamma$ point as in the commensurate twist angle case, such that the location of the $\mathrm{mBZ} \Gamma$ point varies continuously as a function of $\theta$. However, for incommensurate twist angles, a different choice for $\Gamma$ is possible, in principle, and, thus, the wave vector q of the IKS state becomes "gauge dependent." This is consistent with the fact that, for incommensurate twist angles, there is strictly speaking no superlattice translation symmetry. 


\section{B. Generalized Bloch and Lieb-Schulz-Mattis theorems for IKS states}

A defining property of the IKS state, which has circular IVC spiral order, is that it is invariant under the combination of a translation along superlattice vector $\mathbf{a}_{i}$ and a valley- $U(1)$ rotation which shifts the IVC angle by $\mathbf{a}_{i} \cdot \mathbf{q}$. Let us therefore define modified translation operators $\hat{T}_{\mathbf{a}_{i}}^{\prime} \equiv \hat{T}_{\mathbf{a}_{i}} e^{i \mathbf{a}_{i} \cdot \mathbf{q} \tau_{z} / 2}$. Because the IKS state preserves $\hat{T}_{\mathbf{a}_{i}}^{\prime}$, a generalized Bloch theorem applies which states that the single-particle wave functions should satisfy

$$
\psi_{\tilde{\mathbf{k}}}\left(\mathbf{r}+\mathbf{a}_{i}\right)=e^{i \tilde{\mathbf{k}} \cdot \mathbf{a}_{i}} e^{-i \mathbf{a}_{i} \cdot \mathbf{q} \tau_{z} / 2} \psi_{\tilde{\mathbf{k}}}(\mathbf{r}) .
$$

Here, $\tilde{\mathbf{k}}$ is a new "momentum" label restricted to the first $\mathrm{mBZ}$, which differs from the conventional crystal momentum. In particular, $\tilde{\mathbf{k}}$ labels real, physical momenta $\tilde{\mathbf{k}}+$ $\tau_{z} \mathbf{q} / 2$ in the two valleys $\tau_{z}= \pm$. From Eq. (3), it follows that we can write the single-particle wave functions as

$$
\psi_{\tilde{\mathbf{k}}}(\mathbf{r})=e^{i \mathbf{r} \cdot\left(\tilde{\mathbf{k}}-\tau_{z} \mathbf{q} / 2\right)} u_{\tilde{\mathbf{k}}}(\mathbf{r}),
$$

where $u_{\tilde{\mathbf{k}}}(\mathbf{r})$ is the periodic part satisfying $u_{\tilde{\mathbf{k}}}\left(\mathbf{r}+\mathbf{a}_{i}\right)=$ $u_{\tilde{\mathbf{k}}}(\mathbf{r})$. As a result, we can define a Hartree-Fock band structure in the $\mathrm{mBZ}$ for general IKS states, even if the order wave vector $\mathbf{q}$ is incommensurate with the moiré lattice. We note that a similar observation has previously been made for incommensurate circular spin spiral states $[105,106]$.

Another, but closely related, consequence of the $\hat{T}_{\mathbf{a}_{i}}^{\prime}$ symmetry is that the IKS state can have only a nonzero energy gap (ignoring the Goldstone modes) at integer fillings - unless it breaks additional symmetries or develops nontrivial topological order. To see why this is the case, first add a small perturbation of the form

$$
\begin{aligned}
\hat{V}= & h \int d^{2} \mathbf{r}\left[\cos (\mathbf{q} \cdot \mathbf{r}+\alpha) \hat{\psi}^{\dagger}(\mathbf{r}) \tau_{x} \sigma_{x} \hat{\psi}(\mathbf{r})\right. \\
& \left.+\sin (\mathbf{q} \cdot \mathbf{r}+\alpha) \hat{\psi}^{\dagger}(\mathbf{r}) \tau_{y} \sigma_{x} \hat{\psi}(\mathbf{r})\right]
\end{aligned}
$$

to the Hamiltonian. This perturbation preserves $\hat{T}_{\mathbf{a}_{i}}^{\prime}$ but explicitly breaks the valley- $U(1)$ symmetry. As a result, the Goldstone mode of the IKS state acquires a small gap $\Delta_{G} \propto|h|$. Next, we invoke a generalized Lieb-SchulzMattis (LSM) theorem which states that the IKS state with gapped Goldstone modes can have a unique ground state on the cylinder geometry which is separated by a nonzero energy gap from all other states in the spectrum only if the charge per unit cell is integer. To show that such a generalized LSM theorem indeed holds, one can simply use the standard adiabatic flux-insertion argument put forward by Oshikawa [107,108]. The redefinition of the translation symmetry operator by multiplying it with $e^{i \mathbf{a}_{i} \cdot \mathbf{q} \tau_{z} / 2}$ does not change this argument, as the additional factor commutes with the electric-charge $U(1)$ symmetry.
[109] In general, one expects that the gapless states which occur at noninteger fillings (excluding topological order and additional symmetry breaking) will have a vanishing charge gap, meaning that it is possible to create wellseparated particle-hole pairs with arbitrarily small energy.

\section{Structure and energetics of the IKS state}

Microscopically, the $\hat{\mathcal{T}}$-invariant IVC order of the IKS state induces a Kekulé-like pattern on the graphene scale (Fig. 1), with orientation determined by the local IVC angle $\theta_{\text {IVC. }}$. Since the symmetry breaking occurs predominantly within the central bands (see Appendix D, specifically Fig. D. 3), the Kekulé or $\sqrt{3} \times \sqrt{3}$ pattern, which triples the graphene unit cell, is strongest in the $A A$ regions where the flat band wave functions are spatially localized. However, the finite-q character of the IKS state means that the microscopic Kekulé-like patterns differ between different $A A$ regions, as dictated by the combined moire lattice translation and valley- $U(1)$ rotation symmetry $\hat{T}_{\mathbf{a}_{i}}{ }^{\prime}$ noted above [Fig. 3(b)]. For $\mathbf{q}=\mathbf{G}_{1} / 3$, the system forms stripes along the $\mathbf{a}_{2}$ direction where the graphene-scale Kekule pattern is the same. Since the translation-breaking order is purely IVC, with no $\mathbf{- q}$ or higher harmonic components, there is no additional charge reconstruction at the moiré scale (see Sec. VI).

Further insight into the properties of the IKS state can be gained by analyzing its momentum-resolved single-particle density matrix in more detail. Figure 4(b) plots the strength of the IVC in momentum space, showing that it is close to the maximum value $1 / \sqrt{2}$ throughout most of the $\mathrm{mBZ}$. The exceptions are at two lobes in the $\mathrm{mBZ}$, where the electron populations $N_{\tau}(\mathbf{k})$ in the two valleys [Fig. 4(a)] are close to 0 or 2 . The total occupation at each $\mathbf{k}$ is 2 , consistent with an insulating state. The strong momentum dependence of the IKS state sets it apart from previously studied mean-field phases [20]. From our numerics, we find that the same coherence structure is repeated for both spin species. Therefore, henceforth we consider spin to simply be a spectator degree of freedom, an assumption which is further validated by the basis spiral analysis in Sec. IV.

The locations of IVC depletion provide strong clues as to the mechanism underlying IKS formation. In Fig. 4(c), we calculate for each valley the HF spectrum of the lower band of the self-consistent symmetry-preserving semimetal $(\mathrm{SM})$. This captures the major momentum-dependent effects that strain and interactions have on the band structure. The dispersions of the two valleys are related by TRS. All Dirac points lie above $E_{F}$ at $\nu=-2$. Near $\Gamma_{M}$, there is a region of very high energy (red) that coincides with one of the Dirac points. There is also a region of low energy (dark blue) lying in some other region of the $\mathrm{mBZ}$. Because of TRS, the low- and high-energy lobes (indicated by hatched and dotted regions, respectively) in the two valleys are related by $\mathbf{k} \rightarrow-\mathbf{k}$. 

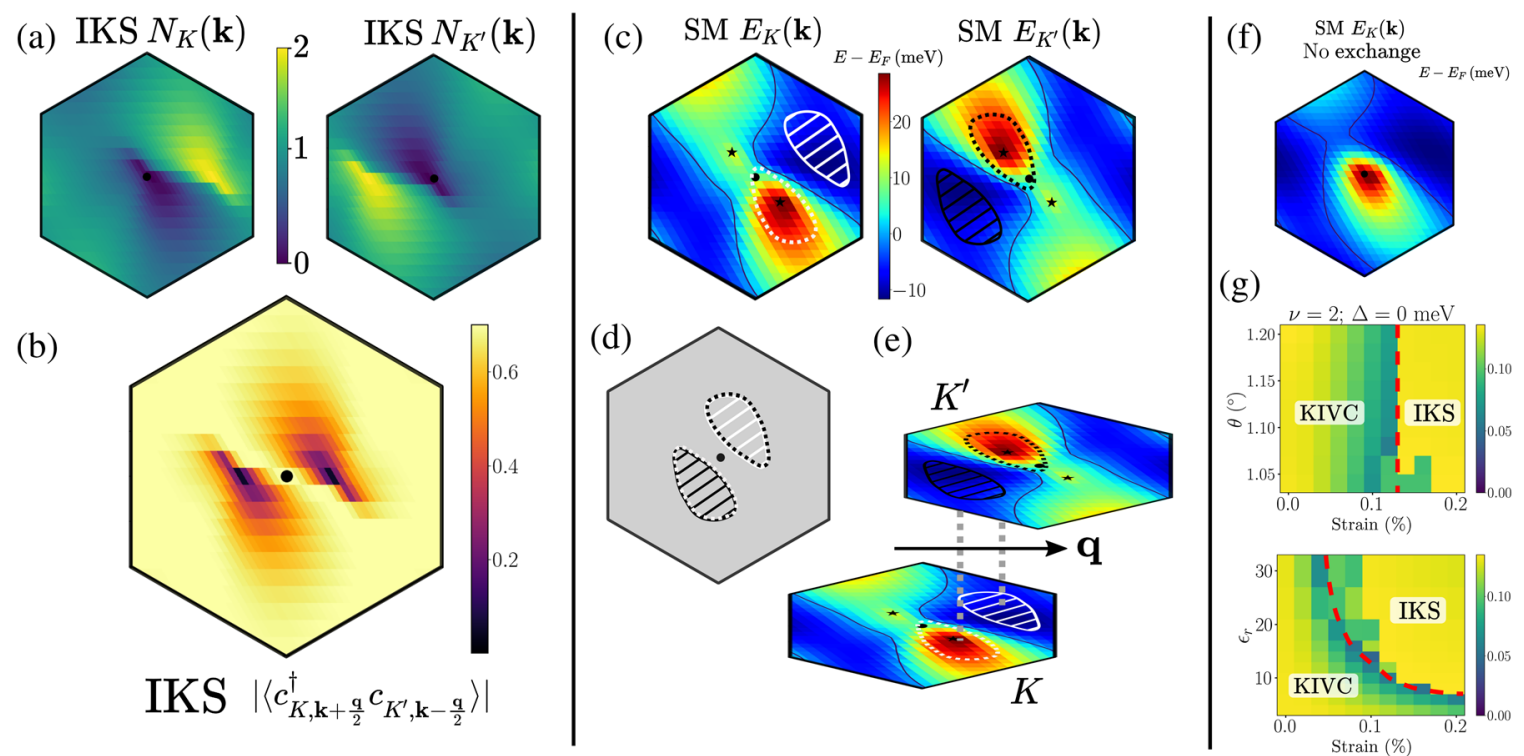

FIG. 4. mBZ-resolved properties of the IKS state at $\nu=-2$. (a) Valley populations of the IKS state. (b) Frobenius norm of the IVC at wave vector $\mathbf{q}=\mathbf{G}_{1} / 3$. System size is $48 \times 16$, strain is $0.2 \%$, substrate is $\Delta=0 \mathrm{meV}$, and NLT is not included. (c) Dispersions of the lower band for the metastable symmetry-preserving self-consistent SM. Hatched (dotted) lobes, situated near low- (high-) energy regions, will be predominantly filled (empty) in the IKS state. Black lines indicate the Fermi surface, and black stars mark Dirac point locations. (d),(e) Schematic construction of the IKS state - a relative momentum boost of the valleys by q allows the lobes to overlap each other. Regions not within the lobes participate strongly in IVC. (f) HF spectrum for the same density matrix used in (c) except that exchange is neglected. (g) $\nu=+2 \mathrm{HF}$ phase diagram in the strain-twist angle plane and the strain-relative permittivity plane. System size is $12 \times 12$.

We now sketch an intuitive picture for how these dispersion features influence the parameters of the IKS order. Figures 4(d) and 4(e) demonstrate that coupling the two valleys at a finite $\mathbf{q}$ can pairwise align a high-energy lobe with a corresponding low-energy lobe in the other valley. In these momentum regions, the system chooses to polarize into the energetically favorable valley [Fig. 4(a)]. Elsewhere, substantial valley hybridization is induced. In this way, the IKS state is able to maximize IVC while respecting the prominent characteristics of the band dispersion. Each $\tilde{\mathbf{k}}$ is equally populated, allowing for an insulating state. Note that attempting to induce IVC at $\mathbf{q}=0$ instead runs into issues - a large portion (approximately $4 \times$ lobe area) of the $\mathrm{mBZ}$ would be unable to participate in the IVC, since the lobes have small overlap. Furthermore, the total electron occupations would vary as a function of $\tilde{\mathbf{k}}$, meaning the state cannot be insulating.

This perspective naturally explains the strong $\mathbf{k}$ dependence of IVC and the slow variation of the IKS energy with q. The somewhat diffuse features of Fig. 4(c) mean that, for nearby $\mathbf{q}$, the locations and shapes of the lobes change only slightly, leading to a small and roughly isotropic wavevector stiffness. A simple estimate for the ideal wave vector $\mathbf{q}_{0}$ can be made by connecting the minimum energy momentum in valley $K^{\prime}$ with the maximum energy peak in valley $K$. The predicted $\mathbf{q}_{0}$ is broadly consistent with HF results of the IKS state for a range of strain angles $\varphi$ (for details, see Appendix D, in particular, Fig. D. 7 [63]).
We emphasize that this scenario opens a gap at $E_{F}$ but, unlike most of the translation-invariant insulators, does not rely on gapping out the Dirac points, which remain high in energy above $E_{F}$. Instead, the k-dependent IVC hybridizes the two valleys at finite $\mathbf{q}$ and pulls the occupied band below the rest of the states [Fig. 5(a)].

We can construct a simple ansatz for the IKS projector in the absence of substrate alignment, which matches the HF numerics extremely well. With $\sigma$ denoting the sublatticepolarized basis, we define two mutually commuting $S U(2)$ Lie algebras $\boldsymbol{\gamma}=\left(\sigma_{x}, \tau_{z} \sigma_{y}, \tau_{z} \sigma_{z}\right)$ and $\boldsymbol{\eta}=\left(\tau_{x} \sigma_{x}, \tau_{y} \sigma_{x}, \tau_{z}\right)$, in terms of which $C=\gamma_{z}$ [21]. We partially fix the gauge by requiring that $\hat{C}_{2 z}$ acts as $\tau_{x} \sigma_{x}$ and $\hat{\mathcal{T}}$ as $\tau_{x} \hat{\mathcal{K}}$-the
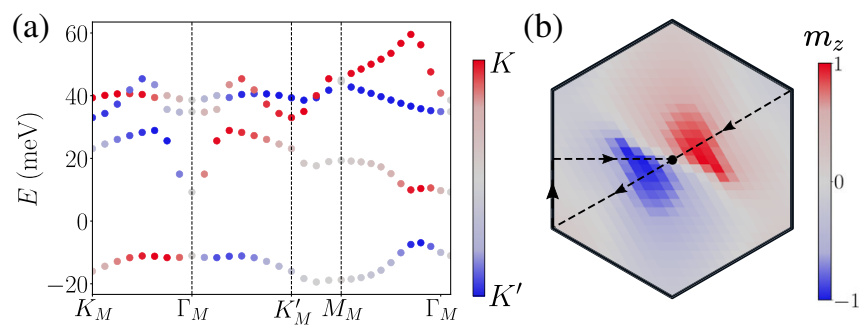

FIG. 5. (a) Band structure of the IKS state at $\nu=-2$, with color indicating the valley polarization of the HF orbitals.) b) $z$ component of the vector $\mathbf{m}(\widetilde{\mathbf{k}})$ in the parametrization of Eq. (6). Dashed lines indicate $\mathrm{mBZ}$ path in (a). In both plots, $\widetilde{\mathbf{k}}$ refers to the valley-dependent boosted momentum $\widetilde{\mathbf{k}}+\tau_{z} \mathbf{q} / 2$. 
remaining gauge freedom acts as $e^{i \varphi_{\mathbf{k}} \tau_{z} \sigma_{z}}$. The spin-singlet IKS state at $\nu=-2$ can then be parametrized by the projector

$$
P_{\nu=-2}(\tilde{\mathbf{k}})=\frac{1}{4}\left(\mathbb{1}+\mathbf{n}_{\tilde{k}} \cdot \boldsymbol{\gamma}\right)\left(\mathbb{1}+\mathbf{m}_{\tilde{k}} \cdot \boldsymbol{\eta}\right),
$$

where $\tilde{\mathbf{k}}$ labels the eigenvalues of $\hat{T}_{\mathbf{a}_{i}}^{\prime}$ as discussed in the previous subsection, the gauge-variant $\mathbf{n}_{\tilde{k}}$ is entirely in the $x-y$ plane, and an identity matrix in spin space is implicit. Across most of the $\mathrm{mBZ}$, the vector $\mathbf{m}_{\tilde{k}}$ lies in plane with a constant angle that can be changed by a global $U(1)_{V}$ rotation. At the lobes, $\mathbf{m}_{\tilde{k}}$ orients toward the poles, reflecting the valley polarization in these momentum regions (recall that $\eta_{z}=\tau_{z}$ ) [Fig. 5(b)]. Since $\gamma_{x}$ and $\gamma_{y}$ in Eq. (6) anticommute with the Chern number $\gamma_{z}$, there is both inter-Chern and intra-Chern IVC in the IKS state with equal magnitude. This implies that the IVC significantly entangles bands with opposite Chern number, in contrast to the usual $U(4) \times U(4)$ ferromagnets found in previous mean-field studies [20]. Importantly, this distinguishes the type of IVC order found here from the uniform $\mathcal{T}$ IVC state of Ref. [20] (which also preserves TRS at even integer fillings). In terms of symmetries, the only difference between the $\mathcal{T}$ IVC and IKS states is that the former is translationally invariant and cannot be made spin singlet by opposite spin rotations in the two valleys. Both states can be made $\hat{C}_{2 z} \hat{\mathcal{T}}$ symmetric by a suitable global valley- $U(1)$ rotation. The IKS projector at $\nu=+2$ can be found by particle-hole conjugation.

The construction outlined above, involving the nesting of features of a parent symmetry-preserving band structure, is suggestive of a weak-coupling instability. However, the $U(1)_{V}$-breaking coherence occurs nearly everywhere in the $\mathrm{mBZ}$, instead of just the lobe boundaries. Furthermore, the Fermi surfaces of the SM or the noninteracting BM model generically bear little relation to the momentum structure in the IKS state. Indeed, both strain and interactions play a vital role in renormalizing the central bands and setting the stage for symmetry-breaking phases - the noninteracting $\mathrm{BM}$ bands have a total bandwidth of approximately $5 \mathrm{meV}$, which broadens to approximately $15 \mathrm{meV}$ in the presence of strain (breaking $\hat{C}_{3 z}$ and shifting the Dirac points up or down), and finally $\gtrsim 50 \mathrm{meV}$ with the inclusion of interactions. Strengthening the Coulomb interaction (by reducing $\epsilon_{r}$ ) favors the strong-coupling states [Fig. 4(g)]. Strain, thus, effectively tunes the system from strong coupling, where Chern-diagonal ferromagnetic states dominate, to intermediate coupling, where other phases (such as the IKS order) emerge that violate the $U(4) \times U(4)$ hierarchy. In the language of Ref. [98], strain does not significantly impact the quality of the "flat-metric condition" (see Appendix D, in particular, Fig. D. 17 [63]), which is used to prove that the uniform $U(4) \times U(4)$ ferromagnet is an exact ground state of the pure interaction
Hamiltonian at all integer $\nu$. Instead, strain substantially increases the dispersion, thereby undermining the validity of the perturbative analysis about the ferromagnetic states and allowing for alternative states such as the IKS to come in. On the other hand, twist angle, which weakly influences the noninteracting central band dispersion, does not have a significant impact on the phase diagram [Fig. 4(g)].

This strong- to weak-coupling crossover can also be viewed through the lens of direct versus exchange energy [110]. The intra-Chern states at small strain are stabilized by exchange, in analogy with quantum Hall ferromagnetism. At larger strains, including just the Hartree piece of the interaction already recreates the key features of the band renormalization, as shown in Fig. 4(f). As verified in Sec. IV, the IKS state is more competitive farther away from charge neutrality, in harmony with the larger Hartree peak (dip) expected for increasing hole (electron) doping [30]. All particles will feel this increased Hartree potential, while exchange effects are applicable only between electrons of the same flavor. We caution though that this direct-exchange dichotomy is not so clear cut in practice-separation of the IKS energy into its components reveals that both Hartree and Fock contributions change significantly with comparable magnitude as a function of q. Also, exchange does significantly perturb the band structure of the self-consistent SM, and its inclusion is necessary to obtain reasonable predictions for $\mathbf{q}_{0}$. This implies that a proper treatment of both terms is required to adequately capture the physics of TBG for realistic parameters (further details in Appendix D, in particular, Figs. D. 8 and D. 11 [63]).

\section{FERROMAGNETIC KEKULÉ SPIRALS AT $\nu= \pm 1$ AND $\nu= \pm 3$}

We now turn to other nonzero fillings, focusing on the same departures from the idealized BM model, fixing NLT as above but exploring the phase structure in the strainsubstrate plane. As we show, IKS order appears to be a ubiquitous feature for relatively modest strain and largely independently of substrate strength.

For $\nu= \pm 3$, a significantly smaller strain $(\epsilon \sim$ $0.02 \%-0.05 \%)$ is able to destabilize the QAH state found upon adding interactions to the unperturbed $\mathrm{BM}$ Hamiltonian [Fig. 6(a)]. Depending on details of the system parameters, it is possible to nucleate an intervening spinvalley polarized nematic semimetal (see Appendix D and, in particular, Fig. D. 2 [63]). At zero substrate potential and strain, an alternative period-doubling stripe which preserves $\hat{C}_{2 z} \hat{\mathcal{T}}$ and $U(1)_{V}$ has also been proposed [51]. With the parameters used in the main text, we find a direct transition from the QAH to an IKS state under increasing strain. The IKS state at $\nu= \pm 3$ has the same symmetry properties as the one at $\nu= \pm 2$, except that it can carry a spin polarization at $U(2)_{K} \times U(2)_{K^{\prime}}$ level (of maximum $1 \mu_{B}$ per moiré cell). For (anti)ferromagnetic Hund's coupling, the spin moments carried by the two valleys (anti)align. The dispersion curve in 


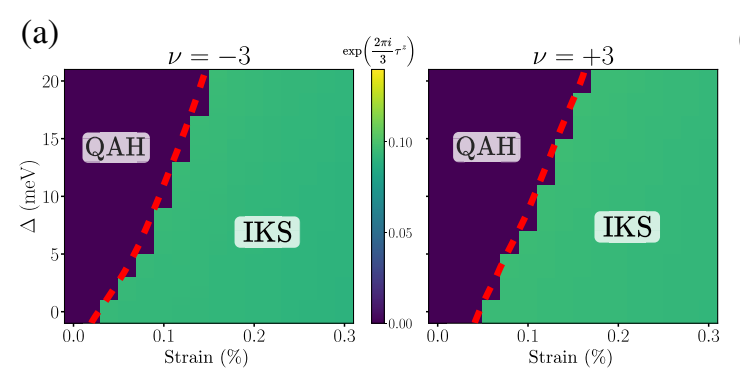

(c)

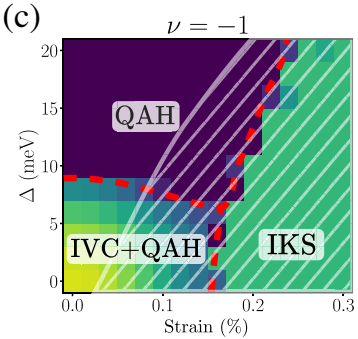

(b)

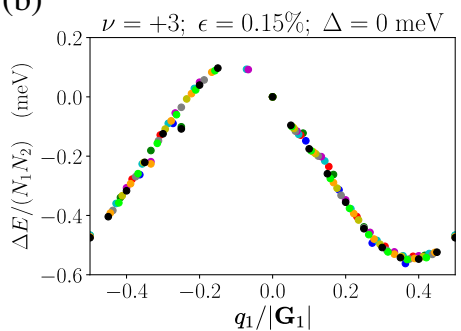

- $11 \times 11$

- $12 \times 12$

- $13 \times 13$

- $14 \times 14$

- $15 \times 15$

- $16 \times 16$

$16 \times 16$
$-\quad 17 \times 17$

- $18 \times 18$

- $19 \times 19$

- $20 \times 20$

FIG. 6. (a) $\nu= \pm 3$ strain-substrate HF phase diagram using the same parameters as Fig. 3 (enlarged view from Fig. 2). (b) Dispersion relation of the IKS state at filling $\nu=+3$ for different system sizes (with $\Delta=0 \mathrm{meV}, \epsilon=0.15 \%$, and no NLT). $\Delta E$ is the energy of the IKS solution at a given wave vector $q \hat{x}$ compared to the energy of the translationally invariant $(q=0)$ QAH state. (c),(d) The same as (a) except at $\nu= \pm 1$ and $\nu=0\left(\epsilon_{r}=15\right)$, again reproducing Fig. 2. Hatched regions denote absence of a charge gap.

Fig. 6(b) is also similar [compare Fig. 3(c)], but it has a deeper minimum and, hence, a larger IVC stiffness, due to the increased renormalization of the bands.

Given the tiny strains required for the IKS to beat the QAH at $\nu= \pm 3$, a natural question that arises is whether the IKS can, in principle, be the ground state at zero strain. We emphasize that there is no fundamental reason that prohibits this scenario from occurring; for strong enough perturbations about the $U(4) \times U(4)$ limit, the strongcoupling insulators can be superseded by states outside the QHFM paradigm. While our Hartree-Fock calculations suggest this is not the case for our choice of parameters, the fact that the IKS can be obtained self-consistently without strain (see Appendix D, in particular, Fig. D. 14 [63], which shows that energy cost is less than $1 \mathrm{meV}$ per unit cell) is a strong indication that the IKS remains a highly competitive state. Altering details of the model Hamiltonian or choosing different parameters (e.g., increasing the relative permittivity) may well tilt the balance in favor of IKS.

At $\nu= \pm 1$, the weak substrate potential region of the $U(1)_{V}$-symmetric QAH phase has nonzero IVC order [15,97,98] [Fig. 6(c)]. The transition from the QAH and QAH + IVC states to the IKS state now occurs at a larger strain $(\epsilon \sim 0.15 \%-0.2 \%)$. The effect of intervalley Hund's terms is similar to that at $\nu= \pm 3$, i.e., a net spin polarization of (0) 1 for (anti)ferromagnetic coupling. In contrast to the other integer fillings, at $\nu= \pm 1$, the IKS state never develops a charge gap. For completeness, in Fig. 6(d), we also present the phase diagram at $\nu=0$, which shows no indications of translation symmetry breaking. The KIVC gives way to a symmetric SM at finite strain [61] and a VH insulator at finite substrate.
All numerically obtained IKS states preserve spinless TRS $\mathcal{T}$, implying that the Chern number $C$ vanishes. This fact is remarkable for the odd fillings, since conventional spin-valley polarized ferromagnets can accommodate only phases with odd $C$. However, recent experiments show the existence of even- $C$ gapped phases extending down to zero magnetic field at odd fillings [30]. One possible route to achieving this is by folding the $\mathrm{mBZ}$ in half and forming period-2 charge order, as theoretically argued by some authors [30]. Each Chern band (a finite sublattice splitting was considered) splits into a $|C|=1$ and 0 miniband, and a variety of different $C$ states can be obtained by selectively polarizing these. Our work proposes a fundamentally distinct scenario, relying instead on IVC to produce the requisite $C=0$ bands and on moiré translation breaking to minimize the energy. The IKS order is agnostic to the presence of substrate alignment and is a natural robust insulating candidate for experiments where strain is often an external confounding factor. Furthermore, as explained in detail in the next section, translation-breaking phases with nonzero Chern number can be obtained by "stacking" a phase with IKS order with other translation-symmetric phases to achieve the requisite band filling. Characterizations of the moiré charge order or strain in the sample of Ref. [30] would help determine which theoretical scenario is operative there.

\section{RELATIONSHIP BETWEEN IKS STATES AT DIFFERENT FILLINGS}

Since the IKS states have similar properties at all nonzero integer fillings, we expect them to be closely related. To make the connection explicit, we consider the 


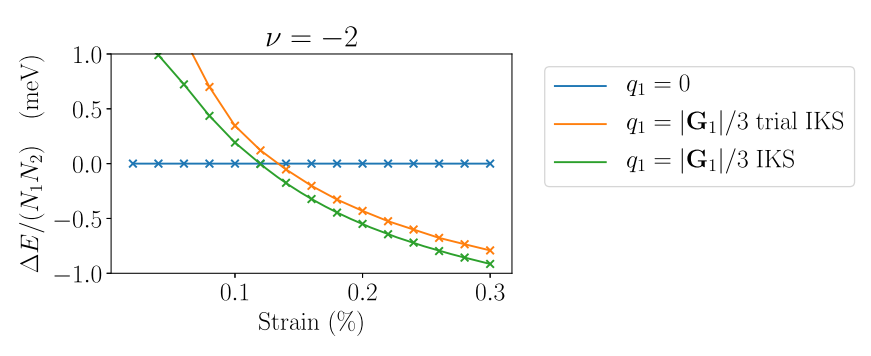

FIG. 7. Comparison of the energies of the translationally invariant state, the IKS state, and the IKS trial state at $\nu=-2$. The IKS trial state is obtained by taking two copies of the IKS HF solution at $\nu=-3$. System size is $12 \times 12$, $\Delta=0 \mathrm{meV}$, and NLT is included.

$|\nu|=3$ IKS state as a "basis spiral." We start with the $\nu=-3$ IKS state with spin polarization enforced for simplicity. In order to construct a $\nu=-2$ IKS, we take two copies of the $\nu=-3$ basis spiral in order to obtain a spin-unpolarized IKS. The same construction is possible at positive filling by particle-hole conjugation. The notion of the $|\nu|=2$ stripe as two copies of the $|\nu|=3$ stripe is consistent with the relative scale of the $U(1)_{V}$-breaking order parameter in Fig. 3(a) being $\sqrt{2}$ times that in Fig. 6(a).

For the $\nu=-1$ IKS state, we note that the translational symmetry breaking is entirely in one spin sector, whereas the other spin sector has the same symmetries as the VH state at $\nu=-2$. This motivates the following construction: We start with the spin-polarized VH state at $\nu=-2$ and add to it the $\nu=-3$ IKS state in the opposite spin sector. Consistent with this fact, the $U(1)_{V}$-breaking order parameter has the same magnitude in the IKS phases at $|\nu|=1$ and 3.

We show in Fig. 7 that the trial states for the $\nu=-2$ IKS state based on this construction have energies that are very close to the self-consistent HF solution at those fillings. We also find that HF simulations using these trial states as initial inputs converge very quickly to the self-consistent IKS ground state at that filling, demonstrating that the trial states have the correct correlations. For $\nu=-1$, the trial state energies are not as close to those of the self-consistent HF IKS state due to the fact the IKS state is not insulating at this filling (see Appendix D [63]).

\section{LANDAU-GINZBURG ANALYSIS}

In this section, we explore the interplay between Kekule spiral states and charge order, by generalizing the LandauGinzburg analysis of Ref. [111]. This has two primary motivations. First, charge order can be detected in a larger variety of experimental probes: For instance, highresolution scanning single-electron transistors (SETs) can detect charge modulation, as can scanning tunneling microscopy (STM) measurements (which can also directly access the moiré-scale-modulated Kekulé distortion characteristic of the IKS state). Second, charge order is more readily pinned by external potentials, and so an IKS state with a subsidiary charge order is likely to respond more strongly to quenched disorder and also to experience stronger commensuration effects.

Our Landau-Ginzburg construction involves the following order parameters: [112]

$$
\begin{gathered}
I_{\mathbf{q}}^{x}=\frac{1}{N} \sum_{\mathbf{k}}\left\langle\hat{\psi}_{\mathbf{k}+\mathbf{q}}^{\dagger} \tau_{x} \sigma_{x} \hat{\psi}_{\mathbf{k}}\right\rangle, \\
I_{\mathbf{q}}^{y}=\frac{1}{N} \sum_{\mathbf{k}}\left\langle\hat{\psi}_{\mathbf{k}+\mathbf{q}}^{\dagger} \tau_{y} \sigma_{x} \hat{\psi}_{\mathbf{k}}\right\rangle, \\
\rho_{\mathbf{q}}=\frac{1}{N} \sum_{\mathbf{k}}\left\langle\hat{\psi}_{\mathbf{k}+\mathbf{q}}^{\dagger} \hat{\psi}_{\mathbf{k}}\right\rangle,
\end{gathered}
$$

where $N$ is the number of moiré unit cells and $\hat{\psi}_{\mathbf{k}, \tau, \sigma}^{\dagger}$ creates an electron in one of the active Chern bands with Chern number $C=\tau_{z} \sigma_{z}$. We also partially fix the gauge by requiring that $\hat{C}_{2 z} \hat{\mathcal{T}}$ acts as $\sigma_{x} \mathcal{K}$ and $\hat{\mathcal{T}}$ as $\tau_{x} \mathcal{K}$. The notation $I$ refers to either "IVC" or "isospin."

Under the symmetries of strained TBG, the order parameters transform as

$$
\begin{aligned}
U(1)_{V}: \mathbf{I}_{\mathbf{q}} & \rightarrow R(\phi) \mathbf{I}_{\mathbf{q}}, \\
\rho_{\mathbf{q}} & \rightarrow \rho_{\mathbf{q}} ; \\
\hat{C}_{2 z}: \mathbf{I}_{\mathbf{q}} & \rightarrow\left(\begin{array}{cc}
1 & \\
& -1
\end{array}\right) \mathbf{I}_{\mathbf{q}}^{*}, \\
\rho_{\mathbf{q}} & \rightarrow \rho_{\mathbf{q}}^{*} ; \\
\hat{\mathcal{T}}: \mathbf{I}_{\mathbf{q}} & \rightarrow \mathbf{I}_{\mathbf{q}}, \\
\rho_{\mathbf{q}} & \rightarrow \rho_{\mathbf{q}},
\end{aligned}
$$

where $\mathbf{I}_{\mathbf{q}}=\left(I_{\mathbf{q}}^{x}, I_{\mathbf{q}}^{y}\right), R(\phi)$ is a $2 \times 2$ rotation matrix, and we use that $\mathbf{I}_{-\mathbf{q}}=\mathbf{I}_{\mathbf{q}}^{*}$ and $\rho_{-\mathbf{q}}=\rho_{\mathbf{q}}^{*}$. Note that both $\hat{C}_{2 z}$ and $\hat{\mathcal{T}}$ act differently on the isospin vector $\mathbf{I}$ than on conventional spin- $1 / 2$ degrees of freedom. An interesting consequence of these unusual symmetry actions is that the bilinear $i \epsilon_{i j} I_{\mathbf{q}}^{i} I_{\mathbf{q}}^{j *}$ with $\epsilon_{i j}=-\epsilon_{j i}$ and $\epsilon_{x y}=1$ respects all symmetries of strained TBG, including superlattice translations.

As a disclaimer, we point out that the Landau-Ginzburg free energy we study below is not invariant under threefold rotations. One justification is that strain breaks $\hat{C}_{3 z}$. However, since the strain is very small, a threefold rotationally symmetric functional (plus small anisotropies) should actually still be an appropriate starting point. Instead, a better justification for a "unidirectional" free energy is that we are interested only in unidirectional physics here. In other words, we can think of our free energy functional as descending from a parent functional 
which is $\hat{C}_{3}$ symmetric but which breaks the threefold rotation symmetry spontaneously. The free energy we write down is then obtained by expanding the parent functional around one of the three valleys.

A Landau-Ginzburg free energy consistent with all the above considerations is given by

$$
\begin{aligned}
F= & \frac{1}{2} r_{\rho}|\rho|^{2}+U_{\rho}|\rho|^{4}+\frac{1}{2} r_{I}|\mathbf{I}|^{2}+U_{I}|\mathbf{I}|^{4} \\
& +\frac{i}{2} r_{x} \epsilon_{i j} I^{i} I^{j *}-U_{x}\left(i \epsilon_{i j} I^{i} I^{j *}\right)^{2} \\
& +\lambda_{1}\left[(\mathbf{I} \cdot \mathbf{I}) \rho^{*}+(\mathbf{I} \cdot \mathbf{I})^{*} \rho\right]+\lambda_{2}|\mathbf{I}|^{2}|\rho|^{2} \\
& +\lambda_{3}|\mathbf{I}|^{2}\left(i \epsilon_{i j} I^{i} I^{j *}\right)+\lambda_{4}|\rho|^{2}\left(i \epsilon_{i j} I^{i} I^{j *}\right),
\end{aligned}
$$

where $\mathbf{I} \equiv I_{\mathbf{q}}$ and $\rho \equiv \rho_{2 \mathbf{q}}$. The terms with coefficients $r_{x}$, $\lambda_{3}$, and $\lambda_{4}$ are not present in the analysis of Ref. [111]. We now argue that, despite the presence of these additional terms, the physical conclusions of Ref. [111] survive. From now on, we normalize the order parameters such that $U_{\rho}=$ $U_{I}=1$ and require that $U_{x}<1$ in order for the free energy to be bounded from below. We also consider the case with $\lambda_{2}=\lambda_{3}=\lambda_{4}=0$, as these terms only quantitatively change the physics we want to discuss here.

As a first step, we choose a coordinate system such that the order parameters can be written as

$$
\begin{gathered}
\mathbf{I}=|\mathbf{I}|\left(\cos \alpha \hat{\mathbf{e}}_{1}+i \sin \alpha \hat{\mathbf{e}}_{2}\right), \\
\rho=|\rho| e^{i \theta},
\end{gathered}
$$

where $\hat{\mathbf{e}}_{i}$ are two orthogonal unit vectors and $0 \leq \alpha \leq \pi / 4$. If $\alpha=0$, then the IVC order is collinear, whereas if $\alpha=\pi / 4$, the Kekulé spiral is perfectly circular, which is what we find in HF simulations. For intermediate values of $\alpha$, the Kekulé spiral has a nonzero eccentricity $\tan \alpha$. Using the above expressions for the order parameters, the free energy can be written as

$$
\begin{aligned}
F= & \frac{1}{2} r_{\rho}|\rho|^{2}+|\rho|^{4}+\frac{1}{2} r_{I}|\mathbf{I}|^{2}+|\mathbf{I}|^{4} \\
& +\frac{1}{2} r_{x}|\mathbf{I}|^{2} \sin 2 \alpha-U_{x}|\mathbf{I}|^{4} \sin ^{2} 2 \alpha \\
& +2 \lambda_{1}|\mathbf{I}|^{2}|\rho| \cos 2 \alpha \cos \theta .
\end{aligned}
$$

Next, we minimize $F$ with respect to $\theta$ in the presence of nonzero $|\mathbf{I}|$. From this, we find that $\theta$ is either 0 or $\pi$, with the optimal value being determined by the sign of $\lambda_{1}$. Note that, for $\theta=0$ or $\pi$, the charge order preserves the $\hat{C}_{2 z}$ symmetry (as does the circular Kekule spiral state).

Now, from minimizing $F$ with respect to $\alpha$, it follows that $\alpha=\pi / 4$ only if $|\rho|=0$. Minimizing $F$ with respect to $|\rho|$ gives us the reverse implication: If $F$ is minimized for $|\rho|=0$, then this implies that $\alpha=\pi / 4$ (and also that $r_{\rho}>0$ ). Combining the above implications, we conclude that there is no charge order if and only if the IKS state is perfectly circular. This interplay between charge order and eccentricity suggests interesting possibilities to experimentally detect the IKS order. For example, let us consider a situation where the TBG sample is strained in a direction orthogonal to the spiral wave vector q. Via the distortion of the graphene bond hoppings, this introduces some nonzero eccentricity for the IKS state and, thus, charge order with half the period of the Kekulé spiral. Conversely, if nonzero charge order is induced via an inhomogeneous electrostatic potential with wave vector $2 \mathbf{q}$, then the IKS state responds by changing the amplitude of the Kekule pattern differently in inequivalent $A A$ regions.

Minimizing $F$ exactly with respect to $\alpha$ is cumbersome, requiring us to minimize the expression:

$$
\frac{1}{2} r_{x} \sin 2 \alpha-U_{x}|\mathbf{I}|^{2} \sin ^{2} 2 \alpha-2\left|\lambda_{1}\right||\rho| \cos 2 \alpha .
$$

Since we are interested in only the parameter regime close to where the circular spiral state is lowest in energy, let us set $2 \alpha=(\pi / 2)+\delta$ and expand in powers of $\delta$. Keeping terms to second order in $\delta$, Eq. (17) becomes

$$
\frac{1}{2} r_{x}\left(1-\frac{1}{2} \delta^{2}\right)-U_{x}|\mathbf{I}|^{2}\left(1-\delta^{2}\right)+2\left|\lambda_{1}\right||\rho| \delta
$$

Minimizing this expression with respect to $\delta$, we find

$$
\delta=\frac{\left|\lambda_{1}\right|}{U_{x}|\mathbf{I}|^{2}-r_{x} / 4}|\rho|,
$$

whence we can write the free energy as

$$
F=\frac{1}{2}\left(r_{\rho}-\frac{4\left|\lambda_{1}\right|^{2}|\mathbf{I}|^{2}}{U_{x}|\mathbf{I}|^{2}-r_{x} / 4}\right)|\rho|^{2}+|\rho|^{4}+\cdots,
$$

where the dots stand for terms which do not involve the charge density (note that if one keeps higher orders of $\delta$, then the coefficient of $|\rho|^{4}$ also receives a correction). It is now clear that there is a second-order phase transition to a phase with nonzero charge order when

$$
r_{\rho}=\frac{4\left|\lambda_{1}\right|^{2}|\mathbf{I}|^{2}}{U_{x}|\mathbf{I}|^{2}-r_{x} / 4} .
$$

As discussed above, in the charge ordered phase the Kekulé spiral necessarily becomes an elliptical spiral. It, thus, follows that by tuning a single parameter it is possible to go from the circular Kekulé spiral phase to an elliptical Kekulé spiral phase via a second-order phase transition. As a direction for future work, it would be interesting to identify experimental knobs that can drive such a transition. 


\section{QUENCHED DISORDER}

For the KIVC state, which occurs at very small strain, quenched disorder is relatively innocuous. The reason is that this state breaks the physical time-reversal symmetry, while physical impurities in graphene are time-reversal symmetric, which implies that they cannot couple as random-field disorder to the KIVC order. The IKS state, on the other hand, preserves time-reversal symmetry and is consequently less protected against disorder. For example, because the IKS state has a Kekulé pattern on every $A A$ region, graphene-scale bond disorder couples to the IVC order as a random field.

Motivated by this observation, we now investigate the effect of bond disorder on the graphene scale in more microscopic detail (we focus on bond disorder for concreteness-the analysis for graphene-scale potential disorder is similar). In particular, we consider the following disorder Hamiltonian:

$$
H_{\mathrm{dis}}=\sum_{\mathbf{R}} \sum_{j=1,2,3} \delta t\left(\mathbf{R}+\boldsymbol{\delta}_{j} / 2\right)\left(c_{\mathbf{R}, A}^{\dagger} c_{\mathbf{R}+\boldsymbol{\delta}_{j}, B}+\text { H.c. }\right),
$$

where $\mathbf{R}$ runs over the positions of the $A$ sites and $\boldsymbol{\delta}_{1}, \boldsymbol{\delta}_{2}$, and $\boldsymbol{\delta}_{3}$ connect each $A$ site to its three neighboring $B$ sites. In the BM basis, the disorder Hamiltonian becomes

$$
H_{\mathrm{dis}}=\frac{1}{A} \sum_{\mathbf{k} \in \mathrm{mBZ}} \sum_{\mathbf{q}} \psi_{\mathbf{k}+\mathbf{q}}^{\dagger} G_{\mathbf{q}}(\mathbf{k}) \psi_{\mathbf{k}}
$$

where $A$ is the area of the sample and

$$
\left[G_{\mathbf{q}}(\mathbf{k})\right]_{\left(\tau^{\prime}, n^{\prime}\right),(\tau, n)}=\left\langle u(\mathbf{k}+\mathbf{q})_{\tau^{\prime}, n^{\prime}}\left|F_{\mathbf{q}}(\mathbf{k})\right| u(\mathbf{k})_{\tau, n}\right\rangle,
$$

Here, $\tau, l$, and $\sigma$, respectively, denote valley, layer, and sublattice, $\left|u(\mathbf{k})_{\tau, n}\right\rangle$ is the periodic part of the BM Bloch states, $\mathbf{G}$ are moiré reciprocal lattice vectors, and $\mathbf{K}$ is the position of the Dirac point corresponding to the $\tau=+$ valley in the graphene Brillouin zone. We also define $\mathbf{q}_{\tau^{\prime} \tau}=$ $\mathbf{q}+\left(\tau^{\prime}-\tau\right) \mathbf{K}$ and $f(\mathbf{k})=\sum_{j=1,2,3} e^{i \boldsymbol{\delta}_{j} \cdot \mathbf{k}}$. Note that at this point we perform an exact unitary transformation, so $n$ and $n^{\prime}$ run over all the $\mathrm{BM}$ bands and not only over the active bands.
Our HF simulations indicate that all IVC order is carried by the active bands, indicating the subspace relevant to studying the order parameter. Let us therefore perform a Schrieffer-Wolff transformation to obtain the effect of $H_{\text {dis }}$ on this subspace. We define $G_{\mathbf{q}}^{i j}(\mathbf{k}) \equiv P^{i} G_{\mathbf{q}}(\mathbf{k}) P^{j}$, where $P^{0}\left(P^{1}\right)$ is the projector onto the active (remote) bands. Assuming that the Fermi energy lies within the active bands, the Schrieffer-Wolff transformed disorder Hamiltonian (up to second order) can be written as follows:

$$
\begin{aligned}
H_{\mathrm{dis}}= & \frac{1}{A} \sum_{\mathbf{k}, \mathbf{q}} \psi_{\mathbf{k}+\mathbf{q}}^{\dagger} G_{\mathbf{q}}^{00}(\mathbf{k}) \psi_{\mathbf{k}} \\
& -\frac{1}{2 A^{2}} \sum_{\mathbf{k}, \mathbf{q}, \mathbf{q}^{\prime}} \sum_{n, n^{\prime}, \tilde{m}} \psi_{n^{\prime}, \mathbf{k}+\mathbf{q}+\mathbf{q}^{\prime}}^{\dagger}\left[G_{\mathbf{q}^{\prime}}^{01}(\mathbf{k}+\mathbf{q})\right]_{n^{\prime} \tilde{m}}\left(\frac{1}{E_{\tilde{m}, \mathbf{k}+\mathbf{q}}-E_{n, \mathbf{k}}}+\frac{1}{E_{\tilde{m}, \mathbf{k}+\mathbf{q}}-E_{n^{\prime}, \mathbf{k}+\mathbf{q}+\mathbf{q}^{\prime}}}\right)\left[G_{\mathbf{q}}^{10}(\mathbf{k})\right]_{\tilde{m} n} \psi_{n, \mathbf{k}},
\end{aligned}
$$

where the sum over $\tilde{m}$ runs over the remote bands and $E_{n, \mathbf{k}}$ are the energies of the BM bands. In what follows, we are interested in only the part of $H_{\text {dis }}$ which couples as a random field to the IKS state, i.e., the part of $H_{\text {dis }}$ which is off diagonal in the valley indices.

Let us first consider the first-order term in $H_{\text {dis }}$. An important observation is that the flat band wave functions vary slowly on the moiré scale. Specifically, the BM eigenvectors satisfy

$$
\sum_{l, \sigma}\left|u(\mathbf{k})_{(\tau, n)}^{l, \sigma, \mathbf{G}}\right|^{2} \approx 0 \quad \text { for }|\mathbf{G}| \gtrsim 2\left|\mathbf{G}_{1}\right|
$$

if $n$ is one of the two flat bands. Here, $\mathbf{G}_{1}$ a basis vector of the moiré reciprocal lattice. This implies that $\left\|G_{\mathbf{q}}^{00}(\mathbf{k})\right\| \approx 0$ if $|\mathbf{q}| \gtrsim 4\left|\mathbf{G}_{1}\right|$. From this, we conclude that the shortwavelength disorder does not couple directly to the IKS order parameter. Furthermore, long-wavelength disorder, which does couple to the IKS state via the first-order term, gets suppressed by a factor $\theta^{2}$. Physically, this is because the density of electrons occupying the central bands is very small.

Because the first-order term in the Schrieffer-Wolff transformed disorder Hamiltonian is inoperative for short-wavelength bond disorder, let us consider the 


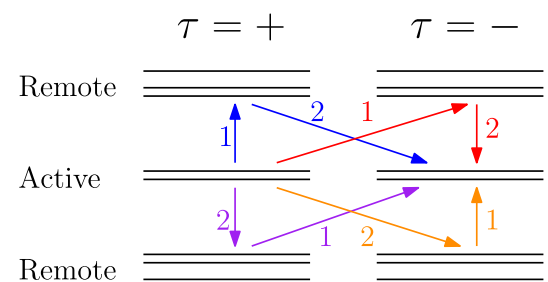

FIG. 8. Virtual processes in second-order perturbation theory which hop an electron from valley $\tau=+$ to valley $\tau=-$ due to quenched random bond disorder.

second-order term next. The relevant second-order processes which hop an electron from valley $\tau=+$ to valley $\tau=-$ are shown schematically in Fig. 8. In the figure, the numbers next to each arrow indicate the order in which the virtual hopping processes take place. There are two processes which involve an intermediate electron in the remote conduction bands and two processes which involve an intermediate hole in the remote valence bands. Note that, in Eq. (26), the energy denominators do not have absolute value signs. This means that the energy denominators involving remote conduction band energies are positive, while energy denominators involving remote valence band energies are negative. This difference in sign comes from the fact that the virtual electron processes generate terms in the second-order Schrieffer-Wolff Hamiltonian of the conventional form $\psi^{\dagger} \psi$, while the virtual hole processes produce terms of the form $\psi \psi^{\dagger}$. The difference in sign, thus, comes from the fermion anticommutation relations.

From Eq. (26), we can estimate the magnitude of the second-order term to be

$$
E_{\mathrm{dis}}^{|\mathbf{q}|>4\left|\mathbf{G}_{1}\right|} \sim \theta^{4} t_{\mathrm{dis}}^{2}\left\langle\Delta^{-1}\right\rangle,
$$

where $t_{\text {dis }}$ is the typical energy scale of the bond disorder and $\left\langle\Delta^{-1}\right\rangle$ is the inverse gap between the active bands and the remote bands averaged over the mini-Brillouin zone. The energy gain of pinning to short-wavelength bond disorder is, thus, reduced by a factor of $\theta^{2} t_{\text {dis }}\left\langle\Delta^{-1}\right\rangle$ compared to the energy gain of pinning to long-wavelength disorder. As a result, for physically relevant disorder strengths we expect the Imry-Ma domains associated with short-wavelength bond disorder to be much larger than the size of the $A A$ regions. For this reason, we ignore shortwavelength disorder and focus only on long-wavelength or moiré-scale random-field disorder. We discuss the effect of moiré-scale quenched disorder on the finite-temperature physics in the following section.

\section{FINITE-TEMPERATURE PHASE TRANSITIONS}

The IKS order breaks not only the valley-charge conservation symmetry, but also superlattice translation and threefold rotation symmetries. It is important to distinguish from the outset the different ways in which the translation and rotation symmetries are broken. In particular, because the IKS states are invariant under a combination of superlattice translation and valley $U(1)$ rotation, only local operators with a nonzero valley charge can detect the translational symmetry breaking. A corollary of this observation is that the translational-symmetry-breaking order is replaced by quasi-long-range order at nonzero temperature and is completely lost once vortices of the IVC order proliferate. The rotational symmetry breaking, on the other hand, can be detected by operators which have zero valley charge. This means that the nematic order (ignoring the explicit symmetry-breaking effects of strain) can persist as true long-range order at nonzero temperatures.

Let us now consider the different possible ways for the IKS order to disappear at finite temperature. We first note that the destruction of IKS order is likely to be driven by the proliferation of fluctuating defects in the order parameter and not by the unbinding of excitons. The reason is that the binding energy of the condensed intervalley coherent excitons is expected to be of the order of the Coulomb scale (approximately $20 \mathrm{meV}$ ), whereas the IKS stiffness extracted from our numerical Hartree-Fock calculations is significantly smaller (approximately $0.4 \mathrm{meV}$ ).

Taking the above considerations into account, there are three different possible scenarios for the IKS order to disappear. In the first scenario, a Berezinskii-KosterlitzThouless (BKT) transition occurs at a temperature $T_{\mathrm{BKT}}$, at which the IKS angle disorders via the unbinding of vortexantivortex pairs. The nematic order, however, persists above $T_{\mathrm{BKT}}$ and disappears at a higher temperature $T_{N}$. [113] In the second scenario, the algebraic IKS order and the nematic order disappear simultaneously via a single phase transition, which most likely is first order. In the third scenario, the nematic order disappears inside the region of the phase diagram with algebraic IKS order, such that $T_{N}<T_{\text {BKT }}$. In Appendix E [63], we show that all these different scenarios can indeed be realized for the special case of commensurate IVC spiral states. In the commensurate case, we find that the finite-temperature physics of IKS states is closely related to that of frustrated or generalized $X Y$ models, which also harbor both quasilong-range order and discrete symmetry breaking [114120]. Exactly which of the three scenarios is realized depends on the ratio $\rho_{s} / \sigma_{\mathrm{DW}}$, where $\rho_{s}$ is the IVC stiffness and $\sigma_{\mathrm{DW}}$ is the domain-wall tension between two different ground states related by $\hat{C}_{3}$. The physical picture which arises in our study of the commensurate models also suggests that the third scenario with $T_{N}<T_{\mathrm{BKT}}$ is excluded in the incommensurate case relevant for TBG. But, regardless of which of the three scenarios occurs experimentally, we expect all transition temperatures to be of the order $T_{\text {BKT }} \lesssim \pi \rho_{s} / 2-7 \mathrm{~K}$.

In our discussion of the finite-temperature phase diagram so far, we have ignored moiré-scale quenched random-field 
disorder. Because TBG is a two-dimensional material, reintroducing quenched disorder, strictly speaking, destroys all ordered phases (breaking both discrete and continuous symmetries). However, if the moiré-scale disorder is sufficiently weak, the finite-temperature phase transitions we discuss for the disorder-free case should still leave detectable imprints on measurable quantities. In particular, for the case of discrete symmetry breaking, it is known that the size of the Imry-Ma domains is exponentially large in $\left(\sigma_{\mathrm{DW}} / h\right)^{2}$, where $\sigma_{\mathrm{DW}}$ again is the domain-wall tension in the ordered state and $h$ is the rms random-field disorder strength [121,122]. For weak disorder, the Imry-Ma domains, thus, are much larger than the relatively small TBG devices currently being used in experiment. In this case, nematicity survives the presence of quenched disorder as "vestigial" order of the IVC spiral state, similarly to what is discussed in Ref. [123] for 3D models of charge density wave order in the context of the cuprate superconductors.

\section{EXPERIMENTS}

We have demonstrated that IKS order is ubiquitous at nonzero integer fillings in the presence of small amounts of heterostrain, largely independent of substrate or fine details of twist angle. We now argue that this fact, combined with the absence of IKS order at charge neutrality in favor of a gapless nematic semimetal, provides a unified explanation of several recent experiments. Evidently, this picture requires us to posit that small heterostrain is inevitably present in typical experimental samples; this is, however, not an unreasonable assumption, particularly given the modest heterostrain needed to stabilize IKS order (which is comparable to experimentally observed strains [80-82]). Before proceeding, we note that in Ref. [124] we extend the Hartree-Fock analysis and investigate the effects of heterostrain upon doping away from integer fillings and including IKS order. By considering the chemical potential variations and the Fermi surface degeneracies, we obtain results in the strained regime that are consistent with the experimentally measured compressibility traces ("cascade" transitions) and Landau fans [1-3,14,25,29,30,88,125-133].

At even integer fillings, the IKS state can be distinguished from the KIVC state by only probing the spin physics. In particular, in a small magnetic field the KIVC state at $\nu= \pm 2$ has a local spin moment of $\lesssim 2 \mu_{B}$ per moire unit cell, [134] whereas the IKS state at $\nu= \pm 2$ has a vanishing local moment. An immediate prediction that follows from this observation is that in samples with negligible strain, which have a strong KIVC gap at neutrality (assuming there is also negligible $h$-BN alignment), the insulators at $\nu= \pm 2$ should have a nonzero local spin moment, whereas strained samples with semimetallic behavior at neutrality should have no (spin or orbital) magnetic moment at $\nu= \pm 2$. By applying a small strain to an initially unstrained sample, one should, therefore, observe a strong first-order transition associated with an abrupt disappearance of the local moment as one enters the IKS phase. If the strain in experiment can be both slowly increased and decreased, hysteretic behaviour should be observed for the local spin moment around the KIVC-IKS transition. We note that Ref. [3] indeed finds evidence for spin-unpolarized insulators at $\nu= \pm 2$ in a sample which is semimetallic at neutrality, which is consistent with the IKS or nematic SM scenario under the assumption that their samples are heterostrained at the approximately $0.1 \%$ $0.2 \%$ level.

At $\nu= \pm 3$, the IKS insulators also have a smaller local magnetic moment than the QAH insulators, which occur in the absence of strain. As both states are spin polarized in a small field, this difference is now due to the large orbital moment of the QAH insulators, which is absent in the IKS insulator. However, the easiest way to distinguish the IKS state from the QAH state is via the transverse or Hall resistance $R_{x y}$. This quantity is zero in the IKS state as dictated by the spinless time-reversal symmetry but takes on a quantized nonzero value in the QAH state. In Refs. [3,15], insulating states are observed at $\nu=+3$ which show Landau fans in magnetotransport measurements that are consistent with a zero Chern number. Given the semimetallic behavior at charge neutrality, we thus expect the samples of Ref. [3,15] to be strained and, therefore, the insulators at $\nu=+3$ to have IKS order.

As discussed in Sec. VIII and Appendix E [63], the nematic order of the IKS state survives at finite temperature. We therefore predict that all insulators at $\nu= \pm 2, \pm 3$ should show strong interaction-induced nematicity, much stronger than what one would naively expect from the small strain present in the sample. This prediction actually fits perfectly with the experimental observations of Ref. [89], where nematicity is observed in the superconducting dome between $\nu=-3$ and $\nu=-2$. Indeed, unless the insulators at integer fillings are separated from the superconducting dome by a strong first-order transition, one would generally expect the insulators and the superconductors to either both be isotropic or both be nematic. In Ref. [124], we show that IKS order persists for a finite range of doping around the integers and survives to temperatures [138] much greater than the experimental $T_{c}$, suggesting that the superconducting dome could indeed inherit physics from the IKS. Furthermore, the ideal ordering wave vector $\mathbf{q}$ [which is naturally soft as illustrated in Fig. 3(d)] changes continuously as a function of density [124], analogous to the evolution of the nematic axis of the superconductor [89]. Such behavior is harder to rationalize for other rotation symmetry-breaking parent states such as stripes. So even without making any assumptions about the nature of the superconducting state, we can interpret the observations of Ref. [89] as indirect evidence for the IKS state.

While the above evidence is reasonable, it remains to a degree circumstantial. A more definitive diagnostic for IKS 
order is possible, in principle, by detecting the Kekule pattern at the $A A$ regions directly using STM or STS. Kekulé order in monolayer graphene induced by mobile adatoms (or substrate vacancies) $[139,140]$ is measured in Ref. [141], whereas Kekulé order induced by large (isotropic) strain $[142,143]$ is observed in Ref. [144]. However, only a very small fraction of the total number of electrons, i.e., those occupying the central bands, participate in the IKS order we find at nonzero integer fillings in TBG. As a result, the signal coming from the graphene-scale $\sqrt{3} \times \sqrt{3}$ Kekulé pattern in the IKS state is significantly smaller than that seen in the above-mentioned monolayer experiments and, hence, could lie below the present-day experimental resolution. [However, the STM studies of Refs. [145,146] are able to detect a Kekulé distortion in monolayer graphene in a high magnetic field at densities that are comparable to those of TBG. The theory here is based on the approximate $S U(4)$ symmetry of the zeroth Landau level, which is close in spirit to the $U(4) \times U(4)$ limit of TBG, but the anisotropies and associated mechanisms are different [147].] In Sec. VI, we discuss how charge order could be induced in the IKS state by changing the eccentricity of the Kekulé spiral. This charge order might be easier to detect experimentally than the Kekulé pattern, for example, by using high-resolution scanning singleelectron transistors made of a carbon nanotube [148,149].

\section{DISCUSSION}

By combining the results obtained in this work at nonzero integer fillings with the findings of Ref. [61] at neutrality, we conclude that by adding a small amount of uniaxial heterostrain to the BM Hamiltonian one obtains from self-consistent Hartree-Fock a global picture of the TBG phase diagram that is consistent with most or even all experimental observations at integer fillings. In particular, at neutrality moderately strained TBG is semimetallic, at $\nu= \pm 1$ it is metallic but with a significantly lower carrier density than the noninteracting BM model due to strong IKS order, and at $\nu= \pm 2, \pm 3$ it becomes an IKS insulator. On top of this, we argue that several other properties of the IKS states (e.g., spin polarization, Chern number, nematicity) fit nicely with many of the experimental observations. The estimated temperatures at which the insulating IKS states should appear (approximately $7 \mathrm{~K}$ ) also agree remarkably well with experiment. Furthermore, as we explain in Ref. [124], the doped descendants of the finite-strain integer orders examined here exhibit Landau fans and compressibility signatures that are consistent with those measured experimentally.

Our results open up several interesting directions for future work. For example, a generalized Pomeranchuk effect is observed in TBG $[150,151]$, which causes highentropy insulators to win over metallic states with increasing temperature. There is at present no theory to fit the IKS state into this Pomeranchuk scenario. For the uniform
QHFM states, several groups have recently calculated the collective mode spectrum [152-155]. In this work, we do not attempt to do this for the IKS states, although it would be helpful to obtain a more complete picture of the low-energy physics at the different integer fillings. Arguably the most interesting direction for future work is to investigate the relation between the IKS states and superconductivity. For example, the KIVC state has recently been argued to be a natural parent state for superconductivity in Refs. $[21,156]$. A future direction would be to adapt this mechanism to the IKS state or investigate whether local KIVC correlations are strong enough to allow the same mechanism to be operative. It would also be interesting to consider if a similar approach to coupling superconductivity via topological terms in Ref. [50] could be generalized to the dominant lobe physics of the IKS. References [126,157] subject TBG to different amounts of Coulomb screening and find that the strengths of insulating and superconducting orders seem to anticorrelate. Any satisfactory theory of superconductivity should be able to explain this anticorrelation.

\section{ACKNOWLEDGMENTS}

We thank Ashvin Vishwanath and Yahui Zhang for interesting discussions on the subtle nature of the translation-symmetry-breaking order in the IKS state. N. B. is supported by a senior postdoctoral research fellowship of the Flanders Research Foundation (FWO). We acknowledge support from the European Research Council under the European Union Horizon 2020 Research and Innovation Program, Grant Agreement No. 804213TMCS, and from EPSRC Grant No. EP/S020527/1. Statement of compliance with EPSRC policy framework on research data: This publication is theoretical work that does not require supporting research data.

[1] Y. Cao, V. Fatemi, A. Demir, S. Fang, S. L. Tomarken, J. Y. Luo, J. D. Sanchez-Yamagishi, K. Watanabe, T. Taniguchi, E. Kaxiras, R. C. Ashoori, and P. Jarillo-Herrero, Correlated Insulator Behaviour at Half-Filling in Magic-Angle Graphene Superlattices, Nature (London) 556, 80 (2018).

[2] Y. Cao, V. Fatemi, S. Fang, K. Watanabe, T. Taniguchi, E. Kaxiras, and P. Jarillo-Herrero, Unconventional Superconductivity in Magic-Angle Graphene Superlattices, Nature (London) 556, 43 (2018).

[3] M. Yankowitz, S. Chen, H. Polshyn, Y. Zhang, K. Watanabe, T. Taniguchi, D. Graf, A. F. Young, and C. R. Dean, Tuning Superconductivity in Twisted Bilayer Graphene, Science 363, 1059 (2019).

[4] X. Lu, P. Stepanov, W. Yang, M. Xie, M. A. Aamir, I. Das, C. Urgell, K. Watanabe, T. Taniguchi, G. Zhang, A. Bachtold, A. H. MacDonald, and D. K. Efetov, Superconductors, Orbital Magnets and Correlated States in Magic-Angle Bilayer Graphene, Nature (London) 574, 653 (2019). 
[5] R. Bistritzer and A.H. MacDonald, Moiré Bands in Twisted Double-Layer Graphene, Proc. Natl. Acad. Sci. U.S.A. 108, 12233 (2011).

[6] J. M. B. Lopes dos Santos, N. M. R. Peres, and A. H. Castro Neto, Graphene Bilayer with a Twist: Electronic Structure, Phys. Rev. Lett. 99, 256802 (2007).

[7] J. M. B. Lopes dos Santos, N. M. R. Peres, and A. H. Castro Neto, Continuum Model of the Twisted Graphene Bilayer, Phys. Rev. B 86, 155449 (2012).

[8] S. Shallcross, S. Sharma, E. Kandelaki, and O. A. Pankratov, Electronic Structure of Turbostratic Graphene, Phys. Rev. B 81, 165105 (2010).

[9] F. Wu, T. Lovorn, E. Tutuc, and A. H. MacDonald, Hubbard Model Physics in Transition Metal Dichalcogenide Moiré Bands, Phys. Rev. Lett. 121, 026402 (2018).

[10] L. Zou, H. C. Po, A. Vishwanath, and T. Senthil, Band Structure of Twisted Bilayer Graphene: Emergent Symmetries, Commensurate Approximants, and Wannier Obstructions, Phys. Rev. B 98, 085435 (2018).

[11] Z. Song, Z. Wang, W. Shi, G. Li, C. Fang, and B. A. Bernevig, All Magic Angles in Twisted Bilayer Graphene Are Topological, Phys. Rev. Lett. 123, 036401 (2019).

[12] J. Kang and O. Vafek, Symmetry, Maximally Localized Wannier States, and a Low-Energy Model for Twisted Bilayer Graphene Narrow Bands, Phys. Rev. X 8, 031088 (2018).

[13] M. Koshino, N. F. Q. Yuan, T. Koretsune, M. Ochi, K. Kuroki, and L. Fu, Maximally Localized Wannier Orbitals and the Extended Hubbard Model for Twisted Bilayer Graphene, Phys. Rev. X 8, 031087 (2018).

[14] M. Serlin, C. L. Tschirhart, H. Polshyn, Y. Zhang, J. Zhu, K. Watanabe, T. Taniguchi, L. Balents, and A. F. Young, Intrinsic Quantized Anomalous Hall Effect in a Moiré Heterostructure, Science 367, 900 (2020).

[15] P. Stepanov, M. Xie, T. Taniguchi, K. Watanabe, X. Lu, A. H. MacDonald, B. A. Bernevig, and D. K. Efetov, Competing Zero-Field Chern Insulators in Superconducting Twisted Bilayer Graphene, Phys. Rev. Lett. 127, 197701 (2021).

[16] B.-B. Chen, Y. Da Liao, Z. Chen, O. Vafek, J. Kang, W. Li, and Z. Y. Meng, Realization of Topological Mott Insulator in a Twisted Bilayer Graphene Lattice Model, Nat. Commun. 12, 5480 (2021).

[17] S. M. Girvin, The Quantum Hall Effect: Novel Excitations and Broken Symmetries, Topological Aspects of Low Dimensional Systems, in Proceedings of the LXIX Les Houches Summer School (Birkhäuser Basel, 1999), 10.1007/3-7643-7393-8_4.

[18] S. L. Sondhi, A. Karlhede, S. A. Kivelson, and E. H. Rezayi, Skyrmions and the Crossover from the Integer to Fractional Quantum Hall Effect at Small Zeeman Energies, Phys. Rev. B 47, 16419 (1993).

[19] K. Moon, H. Mori, Kun Yang, S. M. Girvin, A. H. MacDonald, L. Zheng, D. Yoshioka, and S.-C. Zhang, Spontaneous Interlayer Coherence in Double-Layer Quantum Hall Systems: Charged Vortices and Kosterlitz-Thouless Phase Transitions, Phys. Rev. B 51, 5138 (1995).

[20] N. Bultinck, E. Khalaf, S. Liu, S. Chatterjee, A. Vishwanath, and M. P. Zaletel, Ground State and Hidden Symmetry of
Magic-Angle Graphene at Even Integer Filling, Phys. Rev. X 10, 031034 (2020).

[21] E. Khalaf, S. Chatterjee, N. Bultinck, M. P. Zaletel, and A. Vishwanath, Charged Skyrmions and Topological Origin of Superconductivity in Magic Angle Graphene, Sci. Adv. 7, abf5299 (2021).

[22] J. Kang and O. Vafek, Strong Coupling Phases of Partially Filled Twisted Bilayer Graphene Narrow Bands, Phys. Rev. Lett. 122, 246401 (2019).

[23] Strictly speaking, these are not anomalous, since they emerge only in a finite magnetic field. However, the small fields required (significantly smaller than approximately $10 \mathrm{~T}$ corresponding to one flux per unit cell) suggest that these Chern states remain competitive at zero field.

[24] K. P. Nuckolls, M. Oh, D. Wong, B. Lian, K. Watanabe, T. Taniguchi, B. A. Bernevig, and A. Yazdani, Strongly Correlated Chern Insulators in Magic-Angle Twisted Bilayer Graphene, Nature (London) 588, 610 (2020).

[25] S. Wu, Z. Zhang, K. Watanabe, T. Taniguchi, and E. Y. Andrei, Chern Insulators, van Hove Singularities and Topological Flat Bands in Magic-Angle Twisted Bilayer Graphene, Nat. Mater. 20, 488 (2021).

[26] I. Das, X. Lu, J. Herzog-Arbeitman, Z.-D. Song, K. Watanabe, T. Taniguchi, B. A. Bernevig, and D. K. Efetov, Symmetry Broken Chern Insulators and Magic Series of Rashba-like Landau Level Crossings in Magic Angle Bilayer Graphene, Nat. Phys. 17, 710 (2021).

[27] Yu. Saito, J. Ge, L. Rademaker, K. Watanabe, T. Taniguchi, D. A. Abanin, and A.F. Young, Hofstadter Subband Ferromagnetism and Symmetry-Broken Chern Insulators in Twisted Bilayer Graphene, Nat. Phys. 17, 478 (2021).

[28] Note that, while Ref. [29] reported an anomalous Hall effect at $\nu=+3$, the Hall conductance is not quantized, which is consistent with their samples hosting a gapless state at this filling.

[29] A. L. Sharpe, E. J. Fox, A. W. Barnard, J. Finney, K. Watanabe, T. Taniguchi, M. A. Kastner, and D. Goldhaber-Gordon, Emergent Ferromagnetism near Three-Quarters Filling in Twisted Bilayer Graphene, Science 365, 605 (2019).

[30] A. T. Pierce, Y. Xie, J. M. Park, E. Khalaf, S. H. Lee, Y. Cao, D. E. Parker, P. R. Forrester, S. Chen, K. Watanabe, T. Taniguchi, A. Vishwanath, P. Jarillo-Herrero, and A. Yacoby, Unconventional Sequence of Correlated Chern Insulators in Magic-Angle Twisted Bilayer Graphene, arXiv:2101.04123.

[31] J. Zaanen and O. Gunnarsson, Charged Magnetic Domain Lines and the Magnetism of High- $T_{c}$ Oxides, Phys. Rev. B 40, 7391 (1989).

[32] D. Poilblanc and T. M. Rice, Charged Solitons in the Hartree-Fock Approximation to the Large-u Hubbard Model, Phys. Rev. B 39, 9749 (1989).

[33] M. Inui and P. B. Littlewood, Hartree-Fock Study of the Magnetism in the Single-Band Hubbard Model, Phys. Rev. B 44, 4415 (1991).

[34] T. Giamarchi and C. Lhuillier, Variational Monte Carlo Study of Incommensurate Antiferromagnetic Phases in the Two-Dimensional Hubbard Model, Phys. Rev. B 42, 10641 (1990). 
[35] V. J. Emery and S. A. Kivelson, Frustrated Electronic Phase Separation and High-Temperature Superconductors, Physica (Amsterdam) 209C, 597 (1993).

[36] S. A. Kivelson, E. Fradkin, and V. J. Emery, Electronic Liquid-Crystal Phases of a Doped Mott Insulator, Nature (London) 393, 550 (1998).

[37] V. J. Emery, S. A. Kivelson, and J. M. Tranquada, Stripe Phases in High-Temperature Superconductors, Proc. Natl. Acad. Sci. U.S.A. 96, 8814 (1999).

[38] S. A. Kivelson, I. P. Bindloss, E. Fradkin, V. Oganesyan, J. M. Tranquada, A. Kapitulnik, and C. Howald, How to Detect Fluctuating Stripes in the High-Temperature Superconductors, Rev. Mod. Phys. 75, 1201 (2003).

[39] E. Fradkin, S. A. Kivelson, and J. M. Tranquada, Colloquium: Theory of Intertwined Orders in High Temperature Superconductors, Rev. Mod. Phys. 87, 457 (2015).

[40] B.-X. Zheng, C.-M. Chung, P. Corboz, G. Ehlers, M.-P. Qin, R. M. Noack, H. Shi, S. R. White, S. Zhang, and G. K.-L. Chan, Stripe Order in the Underdoped Region of the Two-Dimensional Hubbard Model, Science 358, 1155 (2017).

[41] E. W. Huang, C. B. Mendl, H.-C. Jiang, B. Moritz, and T. P. Devereaux, Stripe Order from the Perspective of the Hubbard Model, npj Quantum Mater. 3, 22 (2018).

[42] K. Machida, Magnetism in La2CuO4 Based Compounds, Physica (Amsterdam) 158C, 192 (1989).

[43] M. Kato, K. Machida, H. Nakanishi, and M. Fujita, Soliton Lattice Modulation of Incommensurate Spin Density Wave in Two Dimensional Hubbard Model-A Mean Field Study, J. Phys. Soc. Jpn. 59, 1047 (1990).

[44] A. A. Koulakov, M. M. Fogler, and B. I. Shklovskii, Charge Density Wave in Two-Dimensional Electron Liquid in Weak Magnetic Field, Phys. Rev. Lett. 76, 499 (1996).

[45] M. M. Fogler, A. A. Koulakov, and B. I. Shklovskii, Ground State of a Two-Dimensional Electron Liquid in a Weak Magnetic Field, Phys. Rev. B 54, 1853 (1996).

[46] R. Moessner and J. T. Chalker, Exact Results for Interacting Electrons in High Landau Levels, Phys. Rev. B 54, 5006 (1996).

[47] M. M. Fogler, Stripe and Bubble Phases in Quantum Hall Systems (Springer, Berlin, 2002), Vol. 595, pp. 98-138, 10.1007/3-540-45649-X_4.

[48] Y.-Z. You and A. Vishwanath, Superconductivity from Valley Fluctuations and Approximate So(4) Symmetry in a Weak Coupling Theory of Twisted Bilayer Graphene, npj Quantum Mater. 4, 16 (2019).

[49] H. Isobe, N. F. Q. Yuan, and L. Fu, Unconventional Superconductivity and Density Waves in Twisted Bilayer Graphene, Phys. Rev. X 8, 041041 (2018).

[50] M. Christos, S. Sachdev, and M. S. Scheurer, Superconductivity, Correlated Insulators, and Wess-ZuminoWitten Terms in Twisted Bilayer Graphene, Proc. Natl. Acad. Sci. U.S.A. 117, 29543 (2020).

[51] J. Kang and O. Vafek, Non-Abelian Dirac Node Braiding and Near-Degeneracy of Correlated Phases at Odd Integer Filling in Magic-Angle Twisted Bilayer Graphene, Phys. Rev. B 102, 035161 (2020).

[52] Yu.-P. Lin and R. M. Nandkishore, Chiral Twist on the High- $T_{c}$ Phase Diagram in Moiré Heterostructures, Phys. Rev. B 100, 085136 (2019).
[53] Yu.-P. Lin and R. M. Nandkishore, Parquet Renormalization Group Analysis of Weak-Coupling Instabilities with Multiple High-Order van Hove Points inside the Brillouin Zone, Phys. Rev. B 102, 245122 (2020).

[54] M. Christos, S. Sachdev, and M. S. Scheurer, Correlated Insulators, Semimetals, and Superconductivity in Twisted Trilayer Graphene, arXiv:2106.02063.

[55] T. M. R. Wolf, O. Zilberberg, G. Blatter, and J. L. Lado, Spontaneous Valley Spirals in Magnetically Encapsulated Twisted Bilayer Graphene, Phys. Rev. Lett. 126, 056803 (2021).

[56] The spiral state proposed in Ref. [55] relies on large Rashba and Zeeman terms and occurs for twist angles close to $2^{\circ}$.

[57] H. Polshyn, Y. Zhang, M. A. Kumar, T. Soejima, P. Ledwith, K. Watanabe, T. Taniguchi, A. Vishwanath, M.P. Zaletel, and A.F. Young, Topological Charge Density Waves at Half-Integer Filling of a Moiré Superlattice, arXiv:2104.01178.

[58] Z. Bi and L. Fu, Excitonic Density Wave and Spin-Valley Superfluid in Bilayer Transition Metal Dichalcogenide, Nat. Commun. 12, 642 (2021).

[59] Specifically, heterostrain in which one layer is strained relative to the other.

[60] Note that, strictly speaking, superlattice translation symmetry is present only for commensurate twist angles. We return to discuss this point in more detail in Sec. III A.

[61] D. E. Parker, T. Soejima, J. Hauschild, M. P. Zaletel, and N. Bultinck, Strain-Induced Quantum Phase Transitions in Magic Angle Graphene, Phys. Rev. Lett. 127, 027601 (2021).

[62] M. He, Y.-H. Zhang, Y. Li, Z. Fei, K. Watanabe, T. Taniguchi, X. Xu, and M. Yankowitz, Competing Correlated States and Abundant Orbital Magnetism in Twisted Monolayer-Bilayer Graphene, Nat. Commun. 12, 4727 (2021).

[63] See Supplemental Material at http://link.aps.org/ supplemental/10.1103/PhysRevX.11.041063 for details of the interacting strained BM model with nonlocal tunneling, additional Hartree-Fock results, and discussion on the statistical mechanics of commensurate IVC spiral states.

[64] N. N. T. Nam and M. Koshino, Lattice Relaxation and Energy Band Modulation in Twisted Bilayer Graphene, Phys. Rev. B 96, 075311 (2017).

[65] S. Carr, S. Fang, Z. Zhu, and E. Kaxiras, Exact Continuum Model for Low-Energy Electronic States of Twisted Bilayer Graphene, Phys. Rev. Research 1, 013001 (2019).

[66] K. Hejazi, C. Liu, H. Shapourian, X. Chen, and L. Balents, Multiple Topological Transitions in Twisted Bilayer Graphene near the First Magic Angle, Phys. Rev. B 99, 035111 (2019).

[67] G. Tarnopolsky, A. J. Kruchkov, and A. Vishwanath, Origin of Magic Angles in Twisted Bilayer Graphene, Phys. Rev. Lett. 122, 106405 (2019).

[68] J. Liu, J. Liu, and X. Dai, Pseudo Landau Level Representation of Twisted Bilayer Graphene: Band Topology and Implications on the Correlated Insulating Phase, Phys. Rev. B 99, 155415 (2019). 
[69] J. Jung, A. M. DaSilva, A. H. MacDonald, and S. Adam, Origin of Band Gaps in Graphene on Hexagonal Boron Nitride, Nat. Commun. 6, 6308 (2015).

[70] N. Bultinck, S. Chatterjee, and M. P. Zaletel, Mechanism for Anomalous Hall Ferromagnetism in Twisted Bilayer Graphene, Phys. Rev. Lett. 124, 166601 (2020).

[71] Y.-H. Zhang, D. Mao, and T. Senthil, Twisted Bilayer Graphene Aligned with Hexagonal Boron Nitride: Anomalous Hall Effect and a Lattice Model, Phys. Rev. Research 1, 033126 (2019).

[72] D. Mao and T. Senthil, Quasiperiodicity, Band Topology, and Moiré Graphene, Phys. Rev. B 103, 115110 (2021).

[73] J. Shi, J. Zhu, and A. H. MacDonald, Moiré Commensurability and the Quantum Anomalous Hall Effect in Twisted Bilayer Graphene on Hexagonal Boron Nitride, Phys. Rev. B 103, 075122 (2021).

[74] A. Abouelkomsan, Z. Liu, and E. J. Bergholtz, ParticleHole Duality, Emergent Fermi Liquids, and Fractional Chern Insulators in Moiré Flatbands, Phys. Rev. Lett. 124, 106803 (2020).

[75] P. J. Ledwith, G. Tarnopolsky, E. Khalaf, and A. Vishwanath, Fractional Chern Insulator States in Twisted Bilayer Graphene: An Analytical Approach, Phys. Rev. Research 2, 023237 (2020).

[76] C. Repellin and T. Senthil, Chern Bands of Twisted Bilayer Graphene: Fractional Chern Insulators and Spin Phase Transition, Phys. Rev. Research 2, 023238 (2020).

[77] Y. H. Kwan, Y. Hu, S. H. Simon, and S. A. Parameswaran, Exciton Band Topology in Spontaneous Quantum Anomalous Hall Insulators: Applications to Twisted Bilayer Graphene, Phys. Rev. Lett. 126, 137601 (2021).

[78] Y. H. Kwan, Y. Hu, S. H. Simon, and S. A. Parameswaran, Excitonic Fractional Quantum Hall Hierarchy in Moiré Heterostructures, arXiv:2003.11559.

[79] N. Stefanidis and I. Sodemann, Excitonic Laughlin States in Ideal Topological Insulator Flat Bands and Their Possible Presence in Moiré Superlattice Materials, Phys. Rev. B 102, 035158 (2020).

[80] A. Kerelsky, L. J. McGilly, D. M. Kennes, L. Xian, M. Yankowitz, S. Chen, K. Watanabe, T. Taniguchi, J. Hone, C. Dean et al., Maximized Electron Interactions at the Magic Angle in Twisted Bilayer Graphene, Nature (London) 572, 95 (2019).

[81] Y. Choi, J. Kemmer, Y. Peng, A. Thomson, H. Arora, R. Polski, Y. Zhang, H. Ren, J. Alicea, G. Refael et al., Electronic Correlations in Twisted Bilayer Graphene near the Magic Angle, Nat. Phys. 15, 1 (2019).

[82] Y. Xie, B. Lian, B. Jäck, X. Liu, C.-L. Chiu, K. Watanabe, T. Taniguchi, B. A. Bernevig, and A. Yazdani, Spectroscopic Signatures of Many-Body Correlations in Magic Angle Twisted Bilayer Graphene, Nature (London) 572, 101 (2019).

[83] S. Liu, E. Khalaf, J. Y. Lee, and A. Vishwanath, Nematic Topological Semimetal and Insulator in Magic-Angle Bilayer Graphene at Charge Neutrality, Phys. Rev. Research 3, 013033 (2021).

[84] L. Huder, A. Artaud, T. L. Quang, G. T. de Laissardière, A. G. M. Jansen, G. Lapertot, C. Chapelier, and V.T. Renard, Electronic Spectrum of Twisted Graphene Layers under Heterostrain, Phys. Rev. Lett. 120, 156405 (2018).
[85] Z. Bi, N. F. Q. Yuan, and L. Fu, Designing Flat Bands by Strain, Phys. Rev. B 100, 035448 (2019).

[86] G. Cao, Atomistic Studies of Mechanical Properties of Graphene, Polymers 6, 2404 (2014).

[87] Y. H. Kwan, S. A. Parameswaran, and S. L. Sondhi, Twisted Bilayer Graphene in a Parallel Magnetic Field, Phys. Rev. B 101, 205116 (2020).

[88] X. Lu, P. Stepanov, W. Yang, M. Xie, M. A. Aamir, I. Das, C. Urgell, K. Watanabe, T. Taniguchi, G. Zhang et al., Superconductors, Orbital Magnets and Correlated States in Magic-Angle Bilayer Graphene, Nature (London) 574, 653 (2019).

[89] Y. Cao, D. Rodan-Legrain, J. M. Park, N. F. Q. Yuan, K. Watanabe, T. Taniguchi, R. M. Fernandes, L. Fu, and P. Jarillo-Herrero, Nematicity and Competing Orders in Superconducting Magic-Angle Graphene, Science 372, 264 (2021).

[90] S. Fang, S. Carr, Z. Zhu, D. Massatt, and E. Kaxiras, Angle-Dependent Ab Initio Low-Energy Hamiltonians for a Relaxed Twisted Bilayer Graphene Heterostructure, arXiv:1908.00058.

[91] M. Xie and A. H. MacDonald, Weak-Field Hall Resistivity and Spin/Valley Flavor Symmetry Breaking in MAtBG, Phys. Rev. Lett. 127, 196401 (2021).

[92] N. R. Walet and F. Guinea, Lattice Deformation, Low Energy Models and Flat Bands in Twisted Graphene Bilayers, arXiv:1903.00340.

[93] M. Xie and A. H. MacDonald, Nature of the Correlated Insulator States in Twisted Bilayer Graphene, Phys. Rev. Lett. 124, 097601 (2020).

[94] K. N. Kudin, G. E. Scuseria, and E. Cancs, A Black-Box Self-Consistent Field Convergence Algorithm: One Step Closer, J. Chem. Phys. 116, 8255 (2002).

[95] E. Cancès and C. Le Bris, On the Convergence of SCF Algorithms for the Hartree-Fock Equations, ESAIM: M2AN 34, 749 (2000).

[96] T. Cea and F. Guinea, Band Structure and Insulating States Driven by Coulomb Interaction in Twisted Bilayer Graphene, Phys. Rev. B 102, 045107 (2020).

[97] Y. Zhang, K. Jiang, Z. Wang, and F. Zhang, Correlated Insulating Phases of Twisted Bilayer Graphene at Commensurate Filling Fractions: A Hartree-Fock Study, Phys. Rev. B 102, 035136 (2020).

[98] B. Lian, Z.-D. Song, N. Regnault, D. K. Efetov, A. Yazdani, and B. A. Bernevig, TBG IV: Exact Insulator Ground States and Phase Diagram of Twisted Bilayer Graphene, Phys. Rev. B 103, 205414 (2021).

[99] F. Xie, A. Cowsik, Z.-D. Song, B. Lian, B. A. Bernevig, and N. Regnault, TBG VI: An Exact Diagonalization Study of Twisted Bilayer Graphene at Nonzero Integer Fillings, Phys. Rev. B 103, 205416 (2021).

[100] T. Soejima, D. E. Parker, N. Bultinck, J. Hauschild, and M. P. Zaletel, Efficient Simulation of Moiré Materials Using the Density Matrix Renormalization Group, Phys. Rev. B 102, 205111 (2020).

[101] P. Potasz, M. Xie, and A. H. MacDonald, Exact Diagonalization for Magic-Angle Twisted Bilayer Graphene, Phys. Rev. Lett. 127, 147203 (2021).

[102] J. Liu and X. Dai, Theories for the Correlated Insulating States and Quantum Anomalous Hall Effect Phenomena in 
Twisted Bilayer Graphene, Phys. Rev. B 103, 035427 (2021).

[103] G. Shavit, E. Berg, A. Stern, and Y. Oreg, Theory of Correlated Insulators and Superconductivity in Twisted Bilayer Graphene, arXiv:2107.08486.

[104] S. Zhang, X. Lu, and J. Liu, Correlated Insulators, Density Wave States, and Their Nonlinear Optical Response in Magic-Angle Twisted Bilayer Graphene, arXiv: 2109.11441.

[105] L. M. Sandratskii, Energy Band Structure Calculations for Crystals with Spiral Magnetic Structure, Physica Status Solidi (b) 136, 167 (1986).

[106] S. Y. Savrasov, A. Toropova, M. I. Katsnelson, A. I. Lichtenstein, V. Antropov, and G. Kotliar, Electronic Structure and Magnetic Properties of Solids, Z. Kristallogr. 220, 473 (2005).

[107] M. Oshikawa, Commensurability, Excitation Gap, and Topology in Quantum Many-Particle Systems on a Periodic Lattice, Phys. Rev. Lett. 84, 1535 (2000).

[108] M. Oshikawa, Topological Approach to Luttinger's Theorem and the Fermi Surface of a Kondo Lattice, Phys. Rev. Lett. 84, 3370 (2000).

[109] One subtlety, however, is that, in the simplest version of the adiabatic flux-insertion setup, one wants to have the stripes run along the axis of the cylinder, which means that the system has to be compactified in the direction parallel to $\mathbf{q}$. Forcing states with incommensurate $\mathbf{q}$ on the cylinder in this way will cause them to adjust $\mathbf{q}$ to take on the closest commensurate value compatible with the periodic boundary conditions. However, because we can make the cylinder circumference arbitrarily big and, thus, the shift in $\mathbf{q}$ arbitrarily small, this will not change whether or not the system has a gap.

[110] F. Guinea and N. R. Walet, Electrostatic Effects, Band Distortions, and Superconductivity in Twisted Graphene Bilayers, Proc. Natl. Acad. Sci. U.S.A. 115, 13174 (2018).

[111] O. Zachar, S. A. Kivelson, and V. J. Emery, Landau Theory of Stripe Phases in Cuprates and Nickelates, Phys. Rev. B 57, 1422 (1998).

[112] Note that there are other operators we could use instead of $I^{x / y}$. For example, $J^{x / y}=(1 / N) \sum_{\mathbf{k}}\left\langle\hat{\psi}_{\mathbf{k}+\mathbf{q}}^{\dagger} \tau_{x / y} \hat{\psi}_{\mathbf{k}}\right\rangle$ and $K^{x / y}= \pm(1 / N) \sum_{\mathbf{k}}\left\langle\hat{\psi}_{\mathbf{k}+\mathbf{q}}^{\dagger} \tau_{y / x} \sigma_{z} \hat{\psi}_{\mathbf{k}}\right\rangle$ have transform similarly to $\mathbf{I}$. However, using all these operators in the Landau-Ginzburg free energy would not change the conclusions of our analysis-so for simplicity, and because $|\mathbf{I}|=\sqrt{\mathbf{J}^{2}+\mathbf{K}^{2}}$ in the IKS state, we consider only $\mathbf{I}$.

[113] The small but nonzero strain present in TBG will turn this second phase transition at $T_{N}$ into a crossover where the nematic order parameter decreases and saturates at a small but nonzero value with increasing temperature.

[114] D. H. Lee, J. D. Joannopoulos, J. W. Negele, and D. P. Landau, Discrete-Symmetry Breaking and Novel Critical Phenomena in an Antiferromagnetic Planar (XY) Model in Two Dimensions, Phys. Rev. Lett. 52, 433 (1984).

[115] D. H. Lee and G. Grinstein, Strings in Two-Dimensional Classical XY Models, Phys. Rev. Lett. 55, 541 (1985).

[116] D. H. Lee, R. G. Caflisch, J. D. Joannopoulos, and F. Y. Wu, Antiferromagnetic Classical XY Model: A Mean-Field Analysis, Phys. Rev. B 29, 2680 (1984).
[117] E. Granato and J. M. Kosterlitz, Critical Behavior of Coupled XY Models, Phys. Rev. B 33, 4767 (1986).

[118] E. Granato, J. M. Kosterlitz, J. Lee, and M. P. Nightingale, Phase Transitions in Coupled XY-Ising Systems, Phys. Rev. Lett. 66, 1090 (1991).

[119] C. L. Henley, Ordering due to Disorder in a Frustrated Vector Antiferromagnet, Phys. Rev. Lett. 62, 2056 (1989).

[120] P. Chandra, P. Coleman, and A. I. Larkin, Ising Transition in Frustrated Heisenberg Models, Phys. Rev. Lett. 64, 88 (1990).

[121] K. Binder, Random-Field Induced Interface Widths in Ising Systems, Z. Phys. B 50, 343 (1983).

[122] E. T. Seppälä and M. J. Alava, Susceptibility and Percolation in Two-Dimensional Random Field Ising Magnets, Phys. Rev. E 63, 066109 (2001).

[123] L. Nie, G. Tarjus, and S. A. Kivelson, Quenched Disorder and Vestigial Nematicity in the Pseudogap Regime of the Cuprates, Proc. Natl. Acad. Sci. U.S.A. 111, 7980 (2014).

[124] G. Wagner, Y. H. Kwan, N. Bultinck, S. H. Simon, and S. A. Parameswaran, Global Phase Diagram of Twisted Bilayer Graphene above $t_{c}$, arXiv:2109.09749.

[125] J. M. Park, Y. Cao, K. Watanabe, T. Taniguchi, and P. Jarillo-Herrero, Flavour Hunds Coupling, Chern Gaps and Charge Diffusivity in Moir Graphene, Nature (London) 592, 4348 (2021).

[126] P. Stepanov, I. Das, X. Lu, A. Fahimniya, K. Watanabe, T. Taniguchi, F. H. L. Koppens, J. Lischner, L. Levitov, and D. K. Efetov, Untying the Insulating and Superconducting Orders in Magic-Angle Graphene, Nature (London) 583, 375 (2020).

[127] U. Zondiner, A. Rozen, D. Rodan-Legrain, Y. Cao, R. Queiroz, T. Taniguchi, K. Watanabe, Y. Oreg, F. von Oppen, Ady Stern, E. Berg, P. Jarillo-Herrero, and S. Ilani, Cascade of Phase Transitions and Dirac Revivals in Magic-Angle Graphene, Nature (London) 582, 203 (2020).

[128] D. Wong, K. P. Nuckolls, M. Oh, B. Lian, Y. Xie, S. Jeon, K. Watanabe, T. Taniguchi, B. A. Bernevig, and A. Yazdani, Cascade of Electronic Transitions in MagicAngle Twisted Bilayer Graphene, Nature (London) 582, 198 (2020).

[129] J. Kang, B. A. Bernevig, and O. Vafek, Cascades between Light and Heavy Fermions in the Normal State of Magic Angle Twisted Bilayer Graphene, arXiv:2104.01145.

[130] Yu. Saito, F. Yang, J. Ge, X. Liu, T. Taniguchi, K. Watanabe, J. I. A. Li, E. Berg, and A. F. Young, Isospin Pomeranchuk Effect in Twisted Bilayer Graphene, Nature (London) 592, 220 (2021).

[131] A. Rozen, J. M. Park, U. Zondiner, Y. Cao, D. RodanLegrain, T. Taniguchi, K. Watanabe, Y. Oreg, A. Stern, E. Berg et al., Entropic Evidence for a Pomeranchuk Effect in Magic-Angle Graphene, Nature (London) 592, 214 (2021).

[132] S. L. Tomarken, Y. Cao, A. Demir, K. Watanabe, T. Taniguchi, P. Jarillo-Herrero, and R. C. Ashoori, Electronic Compressibility of Magic-Angle Graphene Superlattices, Phys. Rev. Lett. 123, 046601 (2019).

[133] J. Yu, B. A. Foutty, Z. Han, M. E. Barber, Y. Schattner, K. Watanabe, T. Taniguchi, P. Phillips, Z.-X. Shen, S. A. Kivelson, and B.E. Feldman, Correlated Hofstadter Spectrum and Flavor Phase Diagram in Magic Angle Graphene, arXiv:2108.00009. 
[134] Adding a ferromagnetic valley Hund's coupling term to the Hamiltonian induces a magnetic moment of $2 \mu_{B}$ per unit cell for the KIVC state at $\nu= \pm 2$, even in the absence of a magnetic field. If, as is argued in Refs. [135,136], the valley Hund's coupling is antiferromagnetic as a result of, e.g., phonon scattering [137], then a small magnetic field causes the spins in the two valleys to cant, producing a magnetic moment per unit cell which is smaller than $2 \mu_{B}$.

[135] E. Khalaf, P. Ledwith, and A. Vishwanath, Symmetry Constraints on Superconductivity in Twisted Bilayer Graphene: Fractional Vortices, 4e Condensates or Nonunitary Pairing, arXiv:2012.05915.

[136] E. Lake and T. Senthil, Reentrant Superconductivity through a Quantum Lifshitz Transition in Twisted Trilayer Graphene, Phys. Rev. B 104, 174505 (2021).

[137] S. Chatterjee, N. Bultinck, and M. P. Zaletel, Symmetry Breaking and Skyrmionic Transport in Twisted Bilayer Graphene, Phys. Rev. B 101, 165141 (2020).

[138] This refers to the mean-field transition temperature $T_{\mathrm{MF}} \sim 50 \mathrm{~K}$, which is related to exciton binding energies. As discussed in Sec. VIII, there is a lower energy scale $T_{\mathrm{BKT}} \sim 7 \mathrm{~K}$ controlled by the stiffness, above which the phase of the IKS is disordered (though likely still nematic).

[139] V. V. Cheianov, V. I. Falko, O. Syljusen, and B. L. Altshuler, Hidden Kekul Ordering of Adatoms on Graphene, Solid State Commun. 149, 1499 (2009).

[140] V. V. Cheianov, O. Syljuasen, B. L. Altshuler, and V. Fal'ko, Ordered States of Adatoms on Graphene, Phys. Rev. B 80, 233409 (2009).

[141] C. Gutiérrez, C.-J. Kim, L. Brown, T. Schiros, D. Nordlund, E. B. Lochocki, K. M. Shen, J. Park, and A. N. Pasupathy, Imaging Chiral Symmetry Breaking from Kekulé Bond Order in Graphene, Nat. Phys. 12, 950 (2016).

[142] C. A. Marianetti and H. G. Yevick, Failure Mechanisms of Graphene under Tension, Phys. Rev. Lett. 105, 245502 (2010).

[143] S. Sorella, K. Seki, O. O. Brovko, T. Shirakawa, S. Miyakoshi, S. Yunoki, and E. Tosatti, Correlation-Driven Dimerization and Topological Gap Opening in Isotropically Strained Graphene, Phys. Rev. Lett. 121, 066402 (2018).

[144] D. Eom and J.-Y. Koo, Direct Measurement of StrainDriven Kekul Distortion in Graphene and Its Electronic Properties, Nanoscale 12, 19604 (2020).

[145] S.-Yu. Li, Yu. Zhang, L.-J. Yin, and L. He, Scanning Tunneling Microscope Study of Quantum Hall Isospin
Ferromagnetic States in the Zero Landau Level in a Graphene Monolayer, Phys. Rev. B 100, 085437 (2019).

[146] X. Liu, G. Farahi, C.-L. Chiu, Z. Papic, K. Watanabe, T. Taniguchi, M.P. Zaletel, and A. Yazdani, Visualizing Broken Symmetry and Topological Defects in a Quantum Hall Ferromagnet, arXiv:2109.11555.

[147] M. Kharitonov, Phase Diagram for the $\nu=0$ Quantum Hall State in Monolayer Graphene, Phys. Rev. B 85, 155439 (2012).

[148] J. Waissman, M. Honig, S. Pecker, A. Benyamini, A. Hamo, and S. Ilani, Realization of Pristine and Locally Tunable One-Dimensional Electron Systems in Carbon Nanotubes, Nat. Nanotechnol. 8, 569 (2013).

[149] M. Honig, J. A. Sulpizio, J. Drori, A. Joshua, E. Zeldov, and S. Ilani, Local Electrostatic Imaging of Striped Domain Order in LaAlO3/SrTiO3, Nat. Mater. 12, 1112 (2013).

[150] Yu. Saito, F. Yang, J. Ge, X. Liu, K. Watanabe, T. Taniguchi, J. I. A. Li, E. Berg, and A. F. Young, Isospin Pomeranchuk Effect and the Entropy of Collective Excitations in Twisted Bilayer Graphene, Nature (London) 592, 220 (2021).

[151] A. Rozen, J. M. Park, U. Zondiner, Y. Cao, D. RodanLegrain, T. Taniguchi, K. Watanabe, Y. Oreg, A. Stern, E. Berg, P. Jarillo-Herrero, and S. Ilani, Entropic Evidence for a Pomeranchuk Effect in Magic-Angle Graphene, Nature (London) 592, 214 (2021).

[152] Y. H. Kwan, G. Wagner, N. Chakraborty, S. H. Simon, and S. A. Parameswaran, Domain Wall Competition in the Chern Insulating Regime of Twisted Bilayer Graphene, Phys. Rev. B 104, 115404 (2021).

[153] E. Khalaf, N. Bultinck, A. Vishwanath, and M. P. Zaletel, Soft Modes in Magic Angle Twisted Bilayer Graphene, arXiv:2009.14827.

[154] B. A. Bernevig, B. Lian, A. Cowsik, F. Xie, N. Regnault, and Z.-D. Song, TBG V: Exact Analytic Many-Body Excitations in Twisted Bilayer Graphene Coulomb Hamiltonians: Charge Gap, Goldstone Modes and Absence of Cooper Pairing, Phys. Rev. B 103, 205415 (2021).

[155] O. Vafek and J. Kang, Renormalization Group Study of Hidden Symmetry in Twisted Bilayer Graphene with Coulomb Interactions, Phys. Rev. Lett. 125, 257602 (2020).

[156] S. Chatterjee, M. Ippoliti, and M. P. Zaletel, Skyrmion Superconductivity: DMRG Evidence for a Topological Route to Superconductivity, arXiv:2010.01144.

[157] X. Liu, Z. Wang, K. Watanabe, T. Taniguchi, O. Vafek, and J. I. A. Li, Tuning Electron Correlation in Magic-Angle Twisted Bilayer Graphene Using Coulomb Screening, Science 371, 1261 (2021). 